\title{
Intra-night optical variability of $\gamma$-ray detected narrow-line Seyfert1 galaxies
}

\author{
Vineet Ojha ${ }^{1 \star}$, Hum Chand ${ }^{2,1}$, Gopal-Krishna ${ }^{3,1}$ \\ ${ }^{1}$ Aryabhatta Research Institute of Observational Sciences (ARIES), Manora Peak, Nainital - 263002, India. \\ ${ }^{2}$ Department of Physics and Astronomical Sciences, Central University of Himachal Pradesh (CUHP), Dharamshala-176215, India \\ ${ }^{3} U M-D A E$ Centre for Excellence in Basic Sciences, Vidyanagari, Mumbai-400098, India.
}

Accepted - Received —; in original form -

\begin{abstract}
We report the first attempt to systematically characterise intra-night optical variability (INOV) of the rare and enigmatic subset of Narrow-Line Seyfert1 galaxies (NLSy1s), which is marked by detection in the $\gamma$-ray band and is therefore endowed with Doppler boosted relativistic jets, like blazars. However, the central engines in these two types of AGN are thought to operate in different regimes of accretion rate. Our INOV search in a fairly large and unbiased sample of $15 \gamma$-ray NLSy1s was conducted in 36 monitoring sessions, each lasting $\geqslant 3$ hrs. In our analysis, special care has been taken to address the possible effect on the differential light curves, of any variation in the seeing disc during the session, since that might lead to spurious claims of INOV from such AGN due to the possibility of a significant contribution from the host galaxy to the total optical emission. From our observations, a duty cycle (DC) of INOV detection in the $\gamma$-ray NLSy1s is estimated to be around $25 \%$ $30 \%$, which is comparable to that known for blazars. This estimate of DC will probably need an upward revision, once it becomes possible to correct for the dilution of the AGN's nonthermal optical emission by the (much steadier) optical emission contributed not only by the host galaxy but also the nuclear accretion disc in these high Eddington rate accretors. Finally, we also draw attention to the possibility that sharp optical flux changes on sub-hour time scale are less rare for $\gamma$-ray NLSy1s, in comparison to blazars.
\end{abstract}

Key words: surveys - galaxies: active - galaxies: jets $-\gamma$-ray-galaxies: photometry galaxies: Seyfert - gamma-rays: galaxies.

\section{INTRODUCTION}

The diversity of intensity variations across the electromagnetic spectrum is a prominent characteristic of active galactic nuclei (AGNs) and it has been extensively leveraged to probe their emission mechanisms occurring on physical scales that are currently inaccessible to direct imaging by any available technique (e.g., Urry \& Padovani 1995; Wagner \& Witzel 1995; Ulrich et al. 1997; Zensus 1997). AGN variability on minutes to hour-like time scales in the optical waveband is termed as intra-night optical variability (INOV, Gopal-Krishna et al. 1993). Such variations have proved particularly useful for probing and characterising the jet activity in blazars and radioloud narrow-line Seyfert 1 (NLSy1) galaxies, as well as

\footnotetext{
^ E-mail: vineet@aries.res.in, vineetojhabhu@gmail.com
}

for identifying any blazar-like signatures in radio-quiet AGNs (e.g., Miller et al. 1989; Gopal-Krishna et al. 1993, 1995; Jang \& Miller 1995; Heidt \& Wagner 1996; Bai et al. 1999; Romero et al. 1999; Fan et al. 2001; Stalin et al. 2004; Gupta \& Joshi 2005; Carini et al. 2007; Ramírez et al. 2009; Goyal et al. 2012, 2013; Kumar et al. 2017; Paliya 2019; Ojha et al. 2019). In the case of blazars, INOV is generally associated with disturbances within their relativistic jets, e.g., the well-known "shock-in-jet" model (e.g., see Marscher \& Gear 1985; Hughes et al. 1991; Qian et al. 1991; Wagner \& Witzel 1995; Marscher 1996). However, helicity, precession or other geometrical effects associated with the jets (e.g., see Camenzind \& Krockenberger 1992; Gopal-Krishna \& Wiita 1992), perhaps linked to short-term instabilities on the surface of the accretion disc but propagated into the relativistic effects jets, have also been considered as possible mechanisms for INOV in blazars (e.g., 
see Chakrabarti \& Wiita 1993; Mangalam \& Wiita 1993; also, Czerny et al. 2008).

As recently summarised in Gopal-Krishna \& Wiita (2018), prominent among the radio-quiet AGNs covered extensively in INOV studies are radioquiet Seyfert galaxies (Carini et al. 2003), radio-quiet quasars (Gopal-Krishna et al. 2003), and weak emission line quasars (Gopal-Krishna et al. 2013; Chand et al. 2014; Kumar et al. 2015, 2016, 2017). A possible cause for the low-level INOV detections in some such cases is the transient formation of micro-arcsec scale, probably in poorly aligned optical synchrotron jets (see, Gopal-Krishna et al. 2003; Stalin et al. 2004). The possibility that misaligned relativistic jets could be hidden among radio-quiet AGNs has also been noted in the context of NLSy1 galaxies (e.g., Foschini 2011; Berton et al. 2018). Nonetheless, INOV studies of these (low-luminosity) AGNs continue to be sparse and have been limited to a small data set (Miller et al. 2000; Ferrara et al. 2001; Klimek et al. 2004; Liu et al. 2010; Paliya et al. 2013a; Kshama et al. 2017; Ojha et al. 2018; Paliya 2019; Ojha et al. 2020b), even though they were discovered over 3 decades ago (Osterbrock \& Pogge 1985) and basically defined by the rather small width of the optical Balmer emission lines, with full width at half-maximum (FWHM) being $<2000 \mathrm{~km} \mathrm{~s}^{-1}$ for $\mathrm{H} \beta$ (Osterbrock \& Pogge 1985; Goodrich et al. 1989), stronger permitted Fe II, and weak $\left[\mathrm{O}_{I I I}\right]$ emission lines such that the flux ratio of $\left[\mathrm{O}_{I I I}\right]_{\lambda 5007} / H \beta$ is $<3$ (Shuder \& Osterbrock 1981). With some possible exceptions, they also show strong [Fe VII] and [Fe X] emission lines (Pogge 2011, see, however, Cracco et al. 2016). In addition to a soft X-ray spectrum (Boller et al. 1996; Wang et al. 1996; Grupe et al. 1998), they also known to display rapid X-ray (and sometimes even optical) flux variability (e.g., Leighly 1999; Komossa \& Meerschweinchen 2000; Miller et al. 2000; Klimek et al. 2004; Liu et al. 2010; Paliya et al. 2013a; Kshama et al. 2017; Ojha et al. 2019). Observational evidence, as reviewed recently in Paliya (2019), suggests that this class of AGN harbour relatively less massive black holes $\left(10^{6}-10^{8} M_{\odot}\right.$, Grupe \& Mathur 2004; Deo et al. 2006; Peterson 2011) which are accreting at a high fraction of the Eddington rate, in contrast to quasars (e.g., Peterson et al. 2000). Interestingly, NLSy1 galaxies (NLSy1s) exhibit the radio-loud/radio-quiet bimodality, displayed by QSOs, and thus are radio-quiet in most cases (Kellermann et al. 2016, and references). Only about $\sim 7 \%$ of NLSy1s are radio-loud with the radioloudness parameter ${ }^{1} \mathrm{R}_{5 \mathrm{GHz}}>10$ (Komossa et al. 2006; Zhou et al. 2006; Rakshit et al. 2017; Singh \& Chand 2018). For $\mathrm{R}_{5 \mathrm{GHz}}>100$, the fraction drops further to just 2 3 per cent (Komossa et al. 2006, see also, Zhou \& Wang 2002; Yuan et al. 2008).

It may be noted that the inference about NLSy1s having relatively less massive black holes $(\mathrm{BH})$, although not uncontested (e.g., Decarli et al. 2008; Marconi et al. 2008; Calderone et al. 2013; Viswanath et al. 2019), appears

1 Radio-loudness is usually parameterised by the ratio (R) of the rest-frame flux densities at $5 \mathrm{GHz}$ and $4400 \AA$, being $\mathrm{R} \leqslant 10$ and $>10$ for radio-quiet and radio-loud quasars, respectively (e.g. see, Stocke et al. 1992; Visnovsky et al. 1992; Kellermann et al. 1994, 1989). nonetheless to be favoured by the weight of evidence, as summarized in Paliya (2019). A crucial implication then would be that the jet activity in NLSy1s is powered by central engines that operate significantly differently from those in quasars and their blazar subset. It is therefore remarkable that the jetted NLSy1s (see below) and blazars engender very similar manifestations of their relativistic jets, such as superluminal motion (see, e.g., Lister 2018), a doublehumped spectral energy distribution (e.g., Abdo et al. 2009c; Paliya et al. 2013b; Paliya 2019), and violent optical and infrared flux variability (Liu et al. 2010; Itoh et al. 2013; Maune et al. 2013; Paliya et al. 2013a). This makes it especially desirable to search for any contrasting observational properties between blazars and the jetted NLSy1s. In this study, we pursue this objective from the standpoint of rapid optical flux variability, i.e., intranight optical variability (INOV) which is now believed to be a key attribute associated with blazar type AGN (see, e.g., Goyal et al. 2013; Gopal-Krishna \& Wiita 2018; Gopal-Krishna et al. 2019).

It is noteworthy that in the case of NLSy1 galaxies, radio emission from star formation process alone could sometimes lead to a radio-loud classification, even if a relativistic jet is not present (Ganci et al. 2019). Therefore, a conclusive evidence that NLSy1s are capable of ejecting relativistic jets emerged only from the rather unexpected discovery of $\gamma$-ray emission from a handful of NLSy1s, using the FermiLarge Area Telescope (Fermi-LAT) ${ }^{2}$ (Abdo et al. 2009a,b,c; Foschini et al. 2010; Foschini 2011; D'Ammando et al. 2012, 2015; Yao et al. 2015; Paliya et al. 2018; Yang et al. 2018; Yao et al. 2019). Since then, the number of such NLSy1s has slowly increased to just about 20 (Paliya 2019), confirming their rarity. Hereinafter, we shall refer to such AGN as $\gamma$-ray NLSy1.

In fact, almost a decade prior to their detection of NLSy1s in $\gamma$-rays, INOV had already been detected from a few NLSy1s (Miller et al. 2000; Ferrara et al. 2001; Klimek et al. 2004). The first INOV detection in a Fermi/LAT detected NLSy1 galaxy was for J094857.30+002224.0, for which Liu et al. (2010) observed a huge INOV amplitude of $\sim 0.5$-mag. This source is known to exhibit strong optical variability on longer time scales as well (Maune et al. 2013). Nonetheless, as an AGN class, $\gamma$-ray NLSy1s are marked by rather poorly known INOV characteristics, primarily because INOV observations have so far been reported for just $6^{3}$ of them (Liu et al. 2010; Paliya et al. 2013a, 2016; Kshama et al. 2017; Ojha et al. 2019, 2020b). Therefore, the primary aim of the present study is to bridge this knowledge gap, by determining the INOV characteristics of this rare and enigmatic class of AGN. For this, we use a much larger sample, now possible to assemble, and employ a single telescope, as well as a uniform analysis procedure for the entire sample.

The paper is structured as follows. In Sect. 2, we outline the sample selection procedure. Sect. 3 provides details of our intra-night optical monitoring and the data reduction procedure. The statistical analysis is presented in Sect. 4 and

\footnotetext{
2 https://heasarc.gsfc.nasa.gov/docs/heasarc/missions/fermi.html 3 We exclude the NLSy1 galaxy J110223.37+223920.5, with $<3 \sigma$ detection in $\gamma$-rays (Foschini et al. 2015), whose INOV observations are reported by Ojha et al. (2020b).
} 
Table 1. The present sample of $15 \gamma$-ray detected NLSy1 galaxies. Columns are as follows: (1) SDSS name of the sources; (2) R-band magnitude, taken from Monet (1998); (3) emission-line redshift, taken from Paliya et al. (2019), and (4) $R_{1.4 \mathrm{GHz}} \equiv f_{1.4 \mathrm{GHz}} / f_{4400 \AA^{\prime}}$, estimated by taking the rest-frame $1.4 \mathrm{GHz}$ flux density from Paliya (2019) and the optical flux density at rest-frame $4400 \AA$, estimated by fitting the SDSS spectrum, following the procedure described in Rakshit et al. (2017). Note that the values of $R_{1.4 \mathrm{GHz}}$ marked with an ' $\star$ ' are taken from Foschini (2011), except for J164442.53+261913.3 for which the value is taken from Yuan et al. (2008) and J122222.99+041315.9 for which $R_{1.4 \mathrm{GHz}}$ has been estimated using its core flux density of $0.6 \mathrm{Jy}$ at $1.4 \mathrm{GHz}$ from Yuan et al. (2008).

\begin{tabular}{cccr}
\hline $\begin{array}{c}\text { SDSS Name } \\
(1)\end{array}$ & $\begin{array}{c}\text { R-mag } \\
(2)\end{array}$ & $\begin{array}{c}z \\
(3)\end{array}$ & $\begin{array}{c}R_{1.4 G H z} \\
(4)\end{array}$ \\
\hline J032441.20+341045.0 & 13.10 & 0.06 & $318^{\star}$ \\
J084957.98+510829.0 & 17.79 & 0.58 & $4496^{\star}$ \\
J093241.15+530633.8 & 18.82 & 0.60 & 19188 \\
J093712.33+500852.1 & 18.88 & 0.28 & 2007 \\
J094635.07+101706.1 & 18.90 & 1.00 & 11731 \\
J094857.32+002225.6 & 18.17 & 0.58 & $846^{\star}$ \\
J095820.90+322401.6 & 15.51 & 0.53 & 1786 \\
J122222.99+041315.9 & 17.06 & 0.97 & $1534^{\star}$ \\
J130522.75+511640.2 & 15.80 & 0.79 & 509 \\
J144318.56+472556.7 & 17.70 & 0.70 & 1921 \\
J150506.48+032630.8 & 17.72 & 0.41 & $3364^{\star}$ \\
J152039.70+421111.0 & 18.09 & 0.48 & 42908 \\
J164442.53+261913.3 & 16.60 & 0.14 & $447^{\star}$ \\
J211817.40+001316.8 & 18.60 & 0.46 & 12410 \\
J211852.90-073229.3 & 19.48 & 0.26 & 4664 \\
\hline
\end{tabular}

our main results followed by a brief discussion are given in Sect. 5. In Sect. 6, we summarise our main conclusions.

\section{THE SAMPLE}

The present sample is a well-defined, large subset of the compilation of all 22 NLSy1s reported to have a $\gamma$-ray detection (Paliya 2019). Our first filter, namely a $\gamma$-ray detection threshold of $>3 \sigma$, led to the rejection of the sources J124634.65+023809.1 and J142106.00+385522.5 (see, Foschini 2011; Paliya et al. 2018). A third source, J200755.18-443444.3, got excluded because its low declination precludes monitoring from our observatory. Out of the remaining 19 sources, another two, namely J110223.37+223920.5 and J164100.1+345453.0 were rejected since their reported $\gamma$ ray detections have not been confirmed by Foschini et al. (2015) and Ciprini \& Fermi-LAT Collaboration (2018). Note that these two sources are also not contained in the Fermi Large Area Telescope Fourth Source Catalog (Abdollahi et al. 2020). The sixth source to be excluded is J003159.9+093618.0, since Paliya (2019) suggests it to be a probable counterpart of the unidentified $\gamma$-ray source 3FGL J003159+093615. The last source to be excluded is 3C 286 (J133108.3+303032.0); unlike all other $\gamma$-ray detected NLSy1s, its radio spectrum is steep and it has been argued that its $\gamma$-ray emission may substantially originate outside the jet (Berton et al. 2018). The final sample of $15 \gamma$-ray NLSy1s is listed in Table 1.

\section{OBSERVATIONS AND DATA REDUCTION}

\subsection{Photometric monitoring observations}

All $15 \gamma$-ray NLSy1s in our sample were monitored in the broadband filter R, with the 1.3 metre $(\mathrm{m})$ Devasthal Fast Optical Telescope (DFOT) located at Devasthal near Nainital (Sagar et al. 2010) and operated by the Aryabhatta Research Institute of Observational Sciences (ARIES), India. The DFOT is a Ritchey-Chretien (RC) telescope with a fast beam (f/4) and has a pointing accuracy better than 10 arcsec rms. It is equipped with a $2 \mathrm{k} \times 2 \mathrm{k}$ deep thermoelectrically cooled (to about $-85^{\circ} \mathrm{C}$ ) Andor CCD camera, has a pixel size of 13.5 microns, a plate scale of 0.53 arcsec per pixel, giving a field-of-view (FOV) of $\sim 18 \times 18 \mathrm{arcmin}^{2}$ on the sky. The detector chip, having a system rms noise and gain of $7.5 e^{-}$and $2.0 e^{-} \mathrm{ADU}^{-1}$, respectively, was read out at the speed of 1 $\mathrm{MHz}$. Each target AGN was monitored for a duration between 3.0 to 5.5 hours (hrs), as is usual for our INOV campaigns, with the aim to enhance the probability of INOV detection (see Carini 1990). Also, in order to strengthen the INOV statistics, each of our $15 \gamma$-ray NLSy1s was monitored at least in two intranight sessions, the solitary exception being the source J093712.33+500852.1 for which bad weather restricted the monitoring to a single successful session. The exposure time for each science frame was set between 4 and 20 minutes, depending on the brightness of the AGN, the sky transparency, and the lunar phase. The typical median seeing (FWHM of the point spread function (PSF)) during our monitoring sessions ranged between $\sim 2$ - 3 arcsec.

\subsection{Data reduction}

Pre-processing of the raw frames was done by performing the bias subtraction, flat-fielding, and cosmic-ray removal using the standard tasks within the $\mathrm{IRAF}^{4}$ software package. Instrumental magnitudes of the target NLSy1 and the two chosen comparison stars registered in the CCD frames were measured by aperture photometry (Stetson 1987, 1992), using the DAOPHOT II algorithm ${ }^{5}$. A key parameter for photometry is the aperture size, used for measuring the instrumental magnitude and the corresponding signal-tonoise ratio $(\mathrm{S} / \mathrm{N})$ of the individual photometric data points. As reported in Howell (1989), S/N is maximised when the photometric aperture radius is approximately equal to the FWHM of the seeing disc, i.e., Point-Spread-Function (PSF). However, we note that the situation can be more complex while dealing with some relatively nearby AGNs (e.g., two sources in our sample, viz., J032441.20+341045.0 at $\mathrm{z}=0.06$; $\mathrm{J} 164442.53+261913.3$ at $\mathrm{z}=0.14$ ), because a significant contribution to the total flux can come from the underlying host galaxy and hence the relative contributions of the (point-like) AGN and the host galaxy to the aperture photometry can vary significantly as the PSF changes during the session. As emphasised by Cellone et al. (2000), the standard analysis of the differential light curves (DLCs) could then lead to statistically significant, yet spurious claims of INOV for small apertures comparable to the PSF. Keeping this caution in mind,

\footnotetext{
${ }^{4}$ Image Reduction and Analysis Facility (http://iraf.noao.edu/)

5 Dominion Astrophysical Observatory Photometry (http://www.astro.wisc.edu/sirtf/daophot2.pdf)
} 


\section{Ojha et al.}

Table 2. The observational log and basic parameters of the comparison stars used for the sample of $15 \gamma$-ray NLSy1 galaxies. Columns are listed as follows: (1) Target AGN and the comparison stars; (2) date(s) of monitoring; (3) right ascension; (4) declination; (5) SDSS g-band magnitude; (6) SDSS $r$-band magnitude; (7) SDSS ' $g-r$ ' colours. The positions and apparent magnitudes of the sources and their comparison stars were taken from the SDSS DR14 (Abolfathi et al. 2018). Due to the non-availability of the SDSS ' $g-r$ ' colours for the source, J032441.20+341045.0, and its comparison stars (marked by ' $t$ ' in columns 5, 6 \& 7), 'B-R' colours have been used from USNO-A2.0 catalog (Monet 1998) with B-magnitude as 14.50 (target AGN), 15.60 (S1), 16.20 (S2) and R-magnitude as 13.70 (target AGN), 14.40 (S1), 14.40 (S2).

\begin{tabular}{|c|c|c|c|c|c|c|}
\hline $\begin{array}{c}\text { Target AGN and } \\
\text { the comparison stars } \\
\text { (1) }\end{array}$ & $\begin{array}{l}\text { Date(s) of monitoring } \\
\text { (2) }\end{array}$ & $\begin{array}{l}\text { R.A.(J2000) } \\
\text { (hh mm ss) } \\
\text { (3) }\end{array}$ & $\begin{array}{c}\text { Dec.(J2000) } \\
\left(\begin{array}{c}\circ / \prime \prime \\
1 \prime\end{array}\right) \\
(4)\end{array}$ & $\begin{array}{c}g \\
(\mathrm{mag}) \\
(5)\end{array}$ & $\begin{array}{c}r \\
(\mathrm{mag}) \\
(6)\end{array}$ & $\begin{array}{c}g-r \\
(\mathrm{mag}) \\
(7)\end{array}$ \\
\hline J032441.20+341045.0 & 2016 Nov. 22, 23; Dec. 02; 2017 Jan. 03, 04 & 032441.20 & +341045.00 & $\underline{+}^{+}$ & $\amalg^{+}$ & $-^{+}$ \\
\hline S1 & & 032453.68 & +341245.62 & $-^{\dagger}$ & $\leftarrow^{+}$ & $-^{+}$ \\
\hline S2 & & 032453.55 & +341116.58 & $\epsilon^{+}$ & $ـ^{+}$ & $-^{+}$ \\
\hline J084957.98+510829.0 & 2017 Dec. 13, 2019 April 08 & 084957.98 & +510829.04 & 18.92 & 18.28 & 0.64 \\
\hline S1 & & 085012.62 & +510808.03 & 19.45 & 18.06 & 1.39 \\
\hline S2 & & 085003.07 & +510912.23 & 17.82 & 17.09 & 0.73 \\
\hline J093241.15+530633.8 & 2019 Jan. 13, 2020 April 11 & 093241.15 & +530633.79 & 18.90 & 18.84 & 0.06 \\
\hline S1 & 2019 Jan. 13 & 093217.61 & +530148.66 & 19.73 & 18.21 & 1.52 \\
\hline S2 & 2019 Jan. 13 & 093241.51 & +530614.10 & 18.72 & 17.50 & 1.22 \\
\hline S3 & 2020 April 11 & 093212.62 & +530909.46 & 17.86 & 16.82 & 1.04 \\
\hline S4 & 2020 April 11 & 093153.14 & +530130.54 & 16.88 & 16.21 & 0.67 \\
\hline J093712.33+500852.1 & 2019 March 23 & 093712.33 & +500852.14 & 19.53 & 18.79 & 0.74 \\
\hline S1 & & 093801.04 & +500849.80 & 18.36 & 17.84 & 0.52 \\
\hline S2 & & 093634.62 & +50 0956.10 & 18.45 & 17.07 & 1.38 \\
\hline J094635.07+101706.1 & 2019 Dec., 26, 29 & 094635.07 & +10 1706.13 & 19.51 & 19.21 & 0.30 \\
\hline $\mathrm{S} 1$ & 2019 Dec., 26, 29 & 094650.04 & +101013.97 & 18.25 & 17.71 & 0.54 \\
\hline S2 & 2019 Dec., 26 & 094635.29 & +101140.29 & 18.02 & 17.64 & 0.38 \\
\hline S3 & 2019 Dec., 29 & 094704.35 & +101552.01 & 18.72 & 18.03 & 0.69 \\
\hline J094857.32+002225.6 & 2016 Dec. 02; 2017 Dec. 21 & 094857.32 & +002225.56 & 18.59 & 18.43 & 0.16 \\
\hline S1 & & 094836.95 & +002422.55 & 17.69 & 17.28 & 0.41 \\
\hline S2 & & 094837.47 & +00 2037.02 & 17.79 & 16.70 & 1.09 \\
\hline J095820.90+322401.6 & 2019 Jan. 08; Feb. 16 & 095820.90 & +322401.60 & 16.01 & 16.00 & 0.01 \\
\hline $\mathrm{S} 1$ & & 095818.30 & +322834.43 & 15.80 & 15.31 & 0.49 \\
\hline S2 & & 095835.20 & +322819.27 & 15.50 & 15.07 & 0.43 \\
\hline J122222.99+041315.9 & 2017 Jan. 03, 04; Feb. 21, 22; March 04, 24 & 122222.99 & +041315.95 & 17.02 & 16.80 & 0.22 \\
\hline S1 & & 122234.02 & +041321.57 & 18.63 & 17.19 & 1.44 \\
\hline S2 & & 122156.12 & +041515.19 & 17.22 & 16.78 & 0.44 \\
\hline $\mathrm{J} 130522.75+511640.2$ & 2017 April 04; 2019 April 25 & 130522.74 & +511640.26 & 17.29 & 17.10 & 0.19 \\
\hline S1 & 2017 April 04 & 130616.16 & +51 1903.67 & 16.96 & 15.92 & 1.04 \\
\hline S2 & 2017 April 04; 2019 April 25 & 130557.57 & +51 1100.97 & 16.35 & 15.26 & 1.09 \\
\hline S3 & 2019 April 25 & 130544.25 & +510735.85 & 17.88 & 16.42 & 1.46 \\
\hline $\mathrm{J} 144318.56+472556.7$ & 2018 March 11, 23 & 144318.56 & +472556.74 & 18.14 & 18.17 & -0.03 \\
\hline $\mathrm{S} 1$ & & 144337.14 & +472303.03 & 17.51 & 16.82 & 0.69 \\
\hline S2 & & 144319.05 & +471900.98 & 18.03 & 16.75 & 1.28 \\
\hline $\mathrm{J} 150506.48+032630.8$ & 2017 March 25; 2018 April 12 & 150506.48 & +032630.84 & 18.64 & 18.22 & 0.42 \\
\hline S1 & & 150532.05 & +032836.13 & 18.13 & 17.64 & 0.49 \\
\hline $\mathrm{S} 2$ & & 150514.52 & +032456.17 & 17.51 & 17.14 & 0.37 \\
\hline J152039.70+421111.0 & 2019 May 05, 2020 March 22 & 152039.70 & +4211 11.19 & 19.31 & 18.94 & 0.37 \\
\hline $\mathrm{S} 1$ & 2019 May 05 & 152042.81 & +420728.32 & 19.43 & 18.03 & 1.40 \\
\hline S2 & 2019 May 05 & 152120.78 & +420320.45 & 17.51 & 17.09 & 0.42 \\
\hline S3 & 2020 March 22 & 152111.63 & +421145.71 & 17.89 & 17.08 & 0.81 \\
\hline S4 & 2020 March 22 & 152057.17 & +421004.87 & 18.13 & 16.68 & 1.45 \\
\hline $\mathrm{J} 164442.53+261913.3$ & 2017 April 03; 2019 April 26 & 164442.53 & +261913.3 & 18.03 & 17.61 & 0.42 \\
\hline $\mathrm{S} 1$ & & 164520.03 & +262054.55 & 16.56 & 15.89 & 0.67 \\
\hline S2 & & 164434.40 & +261530.27 & 16.28 & 15.80 & 0.48 \\
\hline $\mathrm{J} 211817.40+001316.8$ & 2019 June 07,10 & 211817.40 & +001316.76 & 18.71 & 18.45 & 0.26 \\
\hline S1 & 2019 June 07,10 & 211836.48 & +000835.40 & 18.48 & 17.78 & 0.70 \\
\hline S2 & 2019 June 07 & 211803.72 & +000645.79 & 17.99 & 17.41 & 0.58 \\
\hline S3 & 2019 June 10 & 211810.06 & +001530.61 & 17.99 & 17.63 & 0.36 \\
\hline J211852.90-073229.3 & 2018 Oct. 04; 2019 June 09 & 211852.90 & -073229.34 & 18.78 & 17.96 & 0.82 \\
\hline $\mathrm{S} 1$ & & 211825.68 & -073120.72 & 17.83 & 17.28 & 0.55 \\
\hline S2 & & 211852.33 & -073137.64 & 17.78 & 16.88 & 0.90 \\
\hline
\end{tabular}


Table 3. Observational details and the inferred INOV status for the sample of $15 \gamma$-ray NLSy1 galaxies (photometric aperture radius used $=2 \times$ FWHM). The columns are as follows: (1) SDSS name of the NLSy1; (2) date(s) of the monitoring session(s). Dates inside parentheses are for the longest two sessions which we have used for estimating the INOV duty cycle; (3) duration of the monitoring session; (4) number of data points in the DLCs for the monitoring session; (5) median seeing (FWHM in arcsec) for the session; (6) F-values for the two DLCs (relative to the two comparison stars), based on the F ${ }^{\eta}$-test; (7) INOV status inferred from the two DLCs, using the $\mathrm{F}^{\eta}$-test, with $\mathrm{V}=$ variable (confidence level $\geqslant 99 \%$ ), $\mathrm{PV}=$ probable variable (confidence level between 95-99\%), NV = non-variable (confidence level < 95\%); (8) F-values for the 'NLSy1-reference star' DLC, based on the Fenh-test; (9) INOV status inferred using the $\mathrm{F}_{\text {enh }}$-test; (10) mean photometric error for the two DLCs of the target AGN relative to the two comparison stars; (11) mean amplitude of variability in the two DLCs; and (12) exposure time per frame in the DLC.

\begin{tabular}{|c|c|c|c|c|c|c|c|c|c|c|c|}
\hline $\begin{array}{c}\text { NLSy1s } \\
\text { (SDSS name) } \\
\text { (1) }\end{array}$ & $\begin{array}{c}\text { Date(s) } \\
\text { yyyy.mm.dd } \\
\text { (2) }\end{array}$ & $\begin{array}{c}\mathrm{T} \\
(\mathrm{hr}) \\
(3)\end{array}$ & $\mathrm{N}$ & $\begin{array}{c}\text { Median } \\
\text { FWHM } \\
(\operatorname{arcsec}) \\
(5)\end{array}$ & $\begin{array}{l}F^{\eta} \text {-test } \\
F_{1}^{\eta}, F_{2}^{\eta} \\
(6)\end{array}$ & $\begin{array}{l}\text { INOV } \\
\text { status } \\
99 \% \\
(7)\end{array}$ & $\begin{array}{r}F_{e n h} \text {-test } \\
F_{\text {enh }} \\
\text { (8) }\end{array}$ & $\begin{array}{c}\text { INOV } \\
\text { status } \\
99 \% \\
(9)\end{array}$ & $\begin{array}{c}\sqrt{\left\langle\sigma_{i, e r r}^{2}\right\rangle} \\
\text { (AGN-s) } \\
\text { (10) }\end{array}$ & $\begin{array}{r}\bar{\psi}_{s 1, s 2} \\
(\%) \\
(11)\end{array}$ & $\begin{array}{l}\text { Exposure time } \\
\text { for each science } \\
\text { frame (sec) } \\
\text { (12) }\end{array}$ \\
\hline \multirow[t]{5}{*}{ J032441.20+341045.0 } & $(2016.11 .22)$ & 4.42 & 56 & 2.32 & $14.34,16.13$ & $\mathrm{~V}, \mathrm{~V}$ & 17.39 & $\mathrm{~V}$ & 0.003 & 5.38 & 240 \\
\hline & 2016.11 .23 & 4.27 & 54 & 2.13 & $12.01,10.69$ & $\mathrm{~V}, \mathrm{~V}$ & 05.20 & $\mathrm{~V}$ & 0.003 & 4.07 & 240 \\
\hline & (2016.12.02) & 4.41 & 44 & 2.60 & $85.80,88.73$ & $\mathrm{~V}, \mathrm{~V}$ & 98.29 & $\mathrm{~V}$ & 0.003 & 11.44 & 300 \\
\hline & 2017.01.03 & 3.00 & 39 & 2.47 & $08.14,10.55$ & $\mathrm{~V}, \mathrm{~V}$ & 03.68 & $\mathrm{~V}$ & 0.003 & 4.02 & 240 \\
\hline & 2017.01.04 & 3.39 & 33 & 2.45 & $35.03,35.56$ & $\mathrm{~V}, \mathrm{~V}$ & 17.16 & $\mathrm{~V}$ & 0.003 & 7.49 & 300 \\
\hline \multirow[t]{2}{*}{ J084957.98+510829.0 } & (2017.12.13) & 4.42 & 24 & 2.83 & $00.42,00.51$ & $\mathrm{NV}, \mathrm{NV}$ & 00.77 & NV & 0.033 & - & 600 \\
\hline & (2019.04.08) & 3.04 & 13 & 2.88 & $00.66,00.89$ & $\mathrm{NV}, \mathrm{NV}$ & 00.62 & NV & 0.032 & - & 840 \\
\hline \multirow{2}{*}{ J093241.15+530633.8 } & (2019.01.13) & 3.32 & 17 & 2.86 & $00.80,00.81$ & $\mathrm{NV}, \mathrm{NV}$ & 04.09 & $\mathrm{~V}$ & 0.027 & 6.59 & 720 \\
\hline & (2020.04.11) & 3.39 & 10 & 5.24 & $07.94,08.10$ & $\mathrm{~V}, \mathrm{~V}$ & 07.94 & $\mathrm{~V}$ & 0.046 & 44.64 & 1080 \\
\hline J093712.33+500852.1 & (2019.03.23) & 3.40 & 14 & 2.54 & $00.43,00.33$ & $\mathrm{NV}, \mathrm{NV}$ & 01.46 & NV & 0.025 & - & 900 \\
\hline \multirow[t]{2}{*}{ J094635.07+101706.1 } & (2019.12.26) & 4.03 & 14 & 4.02 & $00.30,00.34$ & $\mathrm{NV}, \mathrm{NV}$ & 02.72 & $\mathrm{NV}$ & 0.045 & - & 1020 \\
\hline & (2019.12.29) & 3.56 & 13 & 3.73 & $00.54,00.42$ & $\mathrm{NV}, \mathrm{NV}$ & 01.64 & NV & 0.041 & - & 900 \\
\hline \multirow[t]{2}{*}{ J094857.32+002225.6 } & (2016.12.02) & 4.15 & 17 & 2.58 & $01.71,01.88$ & $\mathrm{NV}, \mathrm{NV}$ & 10.52 & $\mathrm{~V}$ & 0.017 & 7.95 & 780 \\
\hline & $(2017.12 .21)$ & 5.19 & 33 & 2.24 & $13.95,16.53$ & $\mathrm{~V}, \mathrm{~V}$ & 25.26 & $\mathrm{~V}$ & 0.012 & 16.42 & 540 \\
\hline \multirow[t]{2}{*}{ J095820.90+322401.6 } & (2019.01.08) & 3.22 & 27 & 2.81 & $02.80,03.00$ & $\mathrm{~V}, \mathrm{~V}$ & 01.17 & NV & 0.004 & 3.00 & 360 \\
\hline & $(2019.02 .16)$ & 4.05 & 24 & 2.97 & $21.15,21.86$ & $\mathrm{~V}, \mathrm{~V}$ & 24.90 & $\mathrm{~V}$ & 0.009 & 12.98 & 540 \\
\hline \multirow[t]{6}{*}{$\mathrm{J} 122222.99+041315.9$} & 2017.01 .03 & 3.52 & 17 & 2.38 & $00.62,00.30$ & $\mathrm{NV}, \mathrm{NV}$ & 00.68 & NV & 0.018 & - & 600 \\
\hline & 2017.01.04 & 3.14 & 16 & 2.36 & $00.32,00.37$ & $\mathrm{NV}, \mathrm{NV}$ & 01.99 & NV & 0.014 & - & 720 \\
\hline & 2017.02.21 & 4.44 & 41 & 2.65 & $00.74,00.76$ & $\mathrm{NV}, \mathrm{NV}$ & 02.13 & $\mathrm{~V}$ & 0.020 & 6.36 & 360 \\
\hline & $(2017.02 .22)$ & 5.50 & 50 & 2.59 & $03.98,03.60$ & $\mathrm{~V}, \mathrm{~V}$ & 06.51 & $\mathrm{~V}$ & 0.017 & 13.33 & 360 \\
\hline & (2017.03.04) & 4.93 & 39 & 2.61 & $00.72,00.86$ & $\mathrm{NV}, \mathrm{NV}$ & 01.36 & NV & 0.019 & - & 360 \\
\hline & 2017.03 .24 & 3.94 & 39 & 2.37 & $00.93,00.75$ & $\mathrm{NV}, \mathrm{NV}$ & 01.66 & NV & 0.020 & - & 360 \\
\hline \multirow{2}{*}{$\mathrm{J} 130522.75+511640.2$} & (2017.04.04) & 3.79 & 23 & 2.57 & $00.66,00.70$ & $\mathrm{NV}, \mathrm{NV}$ & 02.94 & PV & 0.012 & - & 600 \\
\hline & (2019.04.25) & 3.11 & 22 & 2.77 & $01.42,01.56$ & $\mathrm{NV}, \mathrm{NV}$ & 04.12 & PV & 0.018 & - & 480 \\
\hline \multirow[t]{2}{*}{$\mathrm{J} 144318.56+472556.7$} & (2018.03.11) & 3.23 & 19 & 3.15 & $00.56,00.54$ & $\mathrm{NV}, \mathrm{NV}$ & 02.59 & NV & 0.022 & - & 480 \\
\hline & (2018.03.23) & 3.08 & 23 & 2.33 & $00.36,00.35$ & $\mathrm{NV}, \mathrm{NV}$ & 01.00 & NV & 0.018 & - & 480 \\
\hline \multirow[t]{2}{*}{$\mathrm{J} 150506.48+032630.8$} & $(2017.03 .25)$ & 5.21 & 41 & 2.08 & $00.60,00.59$ & $\mathrm{NV}, \mathrm{NV}$ & 01.04 & NV & 0.028 & - & 420 \\
\hline & (2018.04.12) & 3.05 & 19 & 2.55 & $00.67,00.63$ & $\mathrm{NV}, \mathrm{NV}$ & 00.84 & NV & 0.032 & - & 480 \\
\hline \multirow[t]{2}{*}{$\mathrm{J} 152039.70+421111.0$} & (2019.05.04) & 3.02 & 11 & 3.08 & $00.56,00.61$ & $\mathrm{NV}, \mathrm{NV}$ & 01.43 & NV & 0.031 & - & 900 \\
\hline & (2020.03.22) & 3.00 & 09 & 4.85 & $01.00,01.00$ & $\mathrm{NV}, \mathrm{NV}$ & 03.69 & NV & 0.034 & - & 900 \\
\hline \multirow[t]{2}{*}{$\mathrm{J} 164442.53+261913.3$} & (2017.04.03) & 4.37 & 37 & 2.50 & $01.44,01.28$ & $\mathrm{NV}, \mathrm{NV}$ & 03.53 & $\mathrm{~V}$ & 0.011 & 5.41 & 420 \\
\hline & (2019.04.26) & 3.22 & 24 & 2.27 & $03.06,03.74$ & $\mathrm{~V}, \mathrm{~V}$ & 06.40 & $\mathrm{~V}$ & 0.011 & 7.50 & 480 \\
\hline \multirow[t]{2}{*}{$\mathrm{J} 211817.40+001316.8$} & (2019.06.07) & 3.31 & 10 & 2.19 & $03.00,03.08$ & NV, NV & 26.26 & $\mathrm{~V}$ & 0.046 & 29.75 & 1200 \\
\hline & $(2019.06 .10)$ & 3.17 & 09 & 2.64 & $16.38,18.42$ & $\mathrm{~V}, \mathrm{~V}$ & 144.97 & $\mathrm{~V}$ & 0.032 & 35.57 & 1200 \\
\hline \multirow[t]{2}{*}{$\mathrm{J} 211852.90-073229.3$} & (2018.10.04) & 4.47 & 13 & 2.81 & $01.87,01.26$ & $\mathrm{NV}, \mathrm{NV}$ & 03.47 & NV & 0.014 & - & 900 \\
\hline & (2019.06.09) & 3.15 & 09 & 2.30 & $06.38,06.55$ & $\mathrm{~V}, \mathrm{~V}$ & 12.31 & V & 0.019 & 17.85 & 1200 \\
\hline
\end{tabular}

we have first estimated the PSF (FWHM) for each CCD frame (by averaging the profiles of 5 bright although clearly unsaturated stars in that frame) and treating the median of those values as the PSF (i.e., FWHM) for that session. The photometry was then performed taking four values of aperture radius, viz., $1 \times$ FWHM, $2 \times$ FWHM, $3 \times$ FWHM and $4 \times$ FWHM. The resulting DLCs of the target AGN were compared with the observed trend of variation of the seeing disc (FWHM) during the session, before proceeding with a quantitative analysis. In practice, this turned out to be an extra cautious approach since, except for three sessions (dated: 2019.04.25, 2019.05.04 and 2020.03.22 see Figs. 3, 4), FWHM remained quite steady, (variations mostly within an arcsec), without showing any systematic trend during the session. Under this circumstance, an aperture radius of $2 \times \mathrm{FWHM}$ is expected to yield fairly reliable DLCs for the target AGN, even if its host galaxy is up to 2-mag brighter than the AGN (see, table 2 of Cellone et al. 2000).

As an additional check for a significant host-galaxy contribution to our aperture photometry, we have fitted the brightness profile of a bright (unsaturated) star, placed at the position of our target AGN. The brightness of the star was varied while keeping the FWHM unchanged. The latter was determined by taking the median of the values measured for nearby 10 bright (unsaturated) stars within the same CCD frame. The best-fit profile obtained was then sub- 
Table 4. The DC and $\bar{\psi}$ of INOV, computed for the present sample of $15 \gamma$-ray NLSy1 galaxies, based on the $F_{e n h}$-test and $F^{\eta}$-test.

\begin{tabular}{lllll}
\hline & \multicolumn{3}{l}{ Based on the $F_{\text {enh }}$-test } & \multicolumn{2}{l}{ Based on the $F^{\eta}$-test } \\
\hline$\gamma$-ray NLSy1s & $\begin{array}{c}{ }^{\star} \mathrm{DC} \\
(\%)\end{array}$ & $\begin{array}{c}{ }^{*} \bar{\psi}^{\dagger} \\
(\%)\end{array}$ & $\begin{array}{c}{ }^{*} \mathrm{DC} \\
(\%)\end{array}$ & $\begin{array}{c}{ }^{\star} \bar{\psi}^{\dagger} \\
(\%)\end{array}$ \\
\hline 39 & 16 & 30 & 17 \\
\hline${ }^{\star}$ Only using the 29 sessions, as explained in Sect. 4.1. \\
${ }^{+}$This is the mean value for all the DLCs belonging to the type ' $\mathrm{V}^{\prime}$.
\end{tabular}

tracted out from the target image, to get the residual image. The counts in the residual image were found to be comparable to the sky background counts for all our sources, except for J032441.20+341045.0 with the highest significance reaching $2.13 \sigma$ for the monitoring session dated 2016-11-23. Note that this AGN is a low-redshift NLSy1 galaxy $(z=0.06)$, and is already known to have a clearly visible host galaxy, as discussed in details by Ojha et al. (2019). This basic trend emerging from the model-fitting is in accord with the findings of the recent search for host galaxy emission for NLSy1 galaxies (Olguín-Iglesias et al. 2020). We note that 9 of our NLSy1s are in fact common to their sample and they could detect host galaxies of just 4 of them, which are also the nearest $(z \lesssim$ $0.6)$ ones in their sample. And this is in spite of their using a far more sensitive telescope (the 8.6-m ESO Very Large Telescope (VLT)) than ours and taking much longer exposures (median 1800 seconds) than we took (median 540 seconds) in our observations for our current sample with the $1.3-\mathrm{m}$ DFOT. Additional comments on some individual cases including this low redshift source are provided in Sect. 5.

For each session, DLCs of the target NLSy1 were derived relative to two (steady) comparison stars which were chosen on the basis of their proximity to the target AGN in brightness, the importance of which for a reliable INOV detection has been highlighted by Howell et al. (1988) and further emphasised in Cellone et al. (2007). For 8 sources of our sample, we were able to find at least one comparison star which falls within $\sim 1$-mag of the target AGN. The magnitude offsets $\left(\Delta m_{R}\right)$ for the remaining sources are also not large, namely 2.0-mag (J093241.15+530633.8), 1.57-mag (J094635.07+101706.1), 1.26-mag (J130522.75+511640.2), 1.29-mag (J144318.60+472557.0), 1.53-mag (J152039.70+421111.00), 1.54-mag (J164442.53+261913.3 and 1.40-mag (J211817.40+001316.8). The coordinates and other parameters of the comparison stars used for the different sessions are given in Table 2 for our entire set of $15 \gamma$-ray NLSy1s. The SDSS $g-r$ colours for the target NLSy1 and their chosen comparison stars are: $<0.82$ and $<$ 1.80 in all cases, with the median values being 0.30 and 0.70 , respectively (column 7 in Table 2). It has been demonstrated by Carini et al. (1992) and Stalin et al. (2004) that colour differences of this order should produce a negligible effect on the DLCs as the atmospheric attenuation varies during a session.

\section{STATISTICAL ANALYSIS}

To search for the presence/absence of INOV in a DLC, we have employed two different forms of the $F$-test proposed by de Diego (2010). These are: (i) the standard F-test (hereafter $F^{\eta}$-test, e.g., see Goyal et al. 2012) and (ii) the powerenhanced $F$-test (hereafter $F_{e n h}$-test, e.g., see de Diego 2014). Detailed description of these two tests is given in our previous papers (Ojha et al. 2019, 2020b, and references therein).

In brief, following Goyal et al. (2012), $F^{\eta}$-test can be written as

$$
F_{1}^{\eta}=\frac{\sigma_{(q-s 1)}^{2}}{\eta^{2}\left\langle\sigma_{q-s 1}^{2}\right\rangle}, \quad F_{2}^{\eta}=\frac{\sigma_{(q-s 2)}^{2}}{\eta^{2}\left\langle\sigma_{q-s 2}^{2}\right\rangle}
$$

where $\sigma_{(q-s 1)}^{2}$ and $\sigma_{(q-s 2)}^{2}$ are the variances with $\left\langle\sigma_{q-s 1}^{2}\right\rangle=\sum_{i=1}^{N} \sigma_{i, \text { err }}^{2}(q-s 1) / N$ and $\left\langle\sigma_{q-s 2}^{2}\right\rangle$ being the mean square (formal) rms errors of the individual data points in the 'target AGN - comparison star1' and 'target AGN - comparison star2' DLCs, respectively. The parameter $\eta$ is the error scaling factor and is taken to be 1.5 from Goyal et al. (2012) (see Ojha et al. 2020b). The DLCs of the target AGN relative to the two comparison stars and the 'star-star' DLCs are displayed in the $2^{\text {nd }}, 3^{\text {rd }}$ and $4^{\text {th }}$ panels from the bottom, in Figs. 1-4, respectively.

For the current study, we have set two critical significance levels, $\alpha=0.01$ and $\alpha=0.05$ which correspond to the confidence levels of $99 \%$ and $95 \%$, respectively. The Fvalues, obtained for individual DLCs using Eq. 1 were compared with the adopted critical $F$-value $\left(F_{c}\right)$, and an NLSy1 is deemed to be variable only if the $F$-values computed for both its DLCs are above the critical $F$ value at $99 \%$ confidence level (hereafter, $\left.F_{\mathcal{C}}(0.99)\right)$. The computed $F^{\eta}$-values and the correspondingly inferred variability status for the 36 sessions devoted to the $15 \gamma$-ray NLSy1s are given in columns 6 and 7 of Table 3.

The $F_{\text {enh }}$-test, as described in Ojha et al. (2020b) can be written as

$$
F_{\mathrm{enh}}=\frac{s_{\mathrm{qso}}^{2}}{s_{\mathrm{stc}}^{2}}, \quad s_{\mathrm{stc}}^{2}=\frac{1}{\left(\sum_{j=1}^{k} N_{j}\right)-k} \sum_{j=1}^{k} \sum_{i=1}^{N_{j}} s_{j, i}^{2}
$$

where $s_{\mathrm{qso}}^{2}$ is the variance of the DLC of the target AGN and the reference star (the one matching better in magnitude to the target AGN out of the two chosen comparison stars), while $s_{\text {stc }}^{2}$ is the stacked variance of the DLCs of the comparison stars and the reference star (de Diego 2014). $N_{j}$ is the number of observations of the $j^{\text {th }}$ star and $k$ is the total number of comparison stars.

The $s_{\mathrm{j}, \mathrm{i}}^{2}$ is the scaled square deviation defined as

$$
s_{j, i}^{2}=\omega_{j}\left(m_{j, i}-\bar{m}_{j}\right)^{2}
$$

where $m_{j, i}$ 's are the differential instrumental magnitudes, and $\bar{m}_{j}$ is the mean differential magnitude of the reference star and the $\mathrm{j}^{\text {th }}$ comparison star. The scaling factor $\omega_{j}$ (see also Joshi et al. 2011) is taken as

$$
\omega_{j}=\frac{\left\langle\sigma_{i, e r r}^{2}(q-r e f)\right\rangle}{\left\langle\sigma_{i, e r r}^{2}\left(s_{j}-r e f\right)\right\rangle} .
$$

The $F_{\text {enh }}$ value is estimated using Eq. 2 and compared with its $F_{c}(0.99)$ and $F_{c}(0.95)$. An NLSy1 is considered variable (V) and probable variable (PV) if the computed values of 'NLSy1-reference star' DLC are found to be as $F_{\text {enh }}>$ $F_{c}(0.99)$ and $F_{\mathcal{C}}(0.95)<F_{\text {enh }} \leqslant F_{c}(0.99)$, respectively. The 
estimated $F_{\text {enh }}$-values and the corresponding variability status for the $15 \gamma$-ray NLSy1s are given in columns 8 and 9 of Table 3.

\subsection{Estimation of the INOV duty cycle and amplitude}

For computing the duty cycle (DC) of INOV in our sample of $15 \gamma$-ray NLSy1s, we have followed the definition given by Romero et al. (1999)

$$
D C=100 \frac{\sum_{j=1}^{n} F_{j}\left(1 / \Delta t_{j}\right)}{\sum_{j=1}^{n}\left(1 / \Delta t_{j}\right)} \text { per cent }
$$

where $\Delta t_{j}=\Delta t_{j, o b s}(1+z)^{-1}$ is the actual duration of the $j^{\text {th }}$ monitoring session, corrected for the target AGN's redshift, $z$ (see details in Ojha et al. 2019, 2020b). $F_{j}$ is set equal to 1 if INOV is detected, otherwise, $F_{j}$ is taken to be zero. Secondly, in order to prevent the DC estimate from getting biased towards more frequently monitored sources in the sample, we have chosen to select just two sessions per source for calculating DC, even though data are available for more sessions (Table 3). The selection of the two sessions was kept unbiased, by basing it on the monitoring duration ( $\mathrm{T}$ ) and thus the longest two sessions were selected, as shown by placing the session dates within parentheses on the panels in Figs. 14 and also in Table 3. The computed values for the sample of $15 \gamma$-ray NLSy1, using the two different statistical tests are tabulated in Table 4.

For estimating the amplitude of INOV $(\psi)$ of the monitored AGN, which quantifies its variation in a given session, we followed the definition given by Heidt \& Wagner (1996)

$$
\psi=\sqrt{\left(A_{\max }-A_{\min }\right)^{2}-2 \sigma^{2}}
$$

with $A_{\min , \max }=$ minimum (maximum) values in the 'target AGN - star' DLC and $\sigma^{2}=\eta^{2}\left\langle\sigma_{q-s}^{2}\right\rangle$, where, $\left\langle\sigma_{q-s}^{2}\right\rangle$ is the mean square (formal) rms errors of individual data points and $\eta=1.5$ (Goyal et al. 2012).

\section{RESULTS AND DISCUSSION}

As mentioned in Sect. 1, the present INOV study based on a sample of $15 \gamma$-ray NLSy1 galaxies represents a substantial advance over the previous reports of INOV properties of this rather poorly understood class of AGN. Those earlier investigations were based on just a handful (6) of AGNs in this class. Recall that just two out of all the NLSy1s currently known to have a confirmed $(>3 \sigma)$ detection in $\gamma$ rays have been left out of the present sample, on grounds well-justified in Sect. 2. Thus, we believe that the INOV characterisation presented here should be fairly representative of this intriguing class of jetted AGN. This characterisation would also enable their systematic comparison with the already well established INOV properties of the blazar type AGN whose relativistic jets are not only more powerful, on the whole, but also thought to form under qualitatively different operating conditions of the central engine (Sect. 1). Another potential difference between these two AGN classes rests on the premise that the jetted NLSy1s represent an earlier stage in the evolution of flatspectrum quasars/blazars (e.g., Mathur 2000; Sulentic et al.
2000; Mathur et al. 2001; Fraix-Burnet et al. 2017; Komossa 2018; Paliya 2019).

Out of the total 36 sessions, INOV was significantly detected by both $\mathrm{F}^{\eta}$-test and the enhanced F-test ( $F_{\text {enh }}$-test) in 12 sessions. For another 5 sessions, the claim of INOV detection rests on the $F_{e n h}$-test alone, while for one session only on the $\mathrm{F}^{\eta}$-test. Being mindful of this, we shall base our present discussion on the (more conservative) $\mathrm{F}^{\eta}$-test. Another reason for this stance is that the available INOV information for large sets of blazars, too, is based on this test (see below). But, before proceeding further, let us revisit the issue, already touched upon in Sect. 3.2, that the optical aperture photometry of the AGN in some of low-z NLSy1s may have been significantly affected by a (varying) contribution from the host galaxy, in case the seeing disc (PSF) varied during the session. As emphasised by Cellone et al. (2000), under such condition some DLCs may show statistically significant INOV which is actually spurious (see Sect. 3.2). As seen from Table 3 and Figs. 1-4, data for 29 out of the total 36 sessions have actually been used here for INOV characterisation (see Sect. 4.1). Statistically significant INOV was detected in 13 of the 29 sessions, based on the $F_{\text {enh }}$-test and in 10 sessions using the $\mathrm{F}^{\eta}$-test. Since only the 10 sessions are used here for INOV statistics, we shall presently focus on their DLCs alone, from the viewpoint of a possible impact of any PSF variations. From Figs. 1-4, it is seen that non-negligible systematic PSF variation occurred in just 4 out of the 10 sessions. Their dates and the target AGNs are: 2020.04.11 (J093241.15+530633.8), 2017.02.22, (J122222.99+041315.9), 2019.06.09 (J211852.90-073229.3) and 2019.06.10 (J211817.40+001316.8). A closer inspection of the DLCs of these 4 sessions shows that, except for the one dated 2017.02.22, PSF actually remained fairly steady over the time span when AGN flux showed variation (Figs. 1, 4 ). Only during the session on 2017-02-22 is the gradient of the DLCs of the target AGN (J122222.99+041315.9) seen to overlap in time with the systematic trend found in the PSF. However, the two gradients are seen to anti-correlate (Fig. 3), which is opposite to what is expected in case the aperture photometric measurements were getting significantly contaminated by the underlying galaxy (see, Cellone et al. 2000). Even otherwise, any contamination from the host galaxy can not be significant for this AGN, since its large redshift ( $z=0.97$, Table 1$)$ implies a high intrinsic optical luminosity, enough to swamp the host galaxy. Thus, to sum up, it can be asserted that the inferred INOV detection in neither of the 10 sessions is spurious, and this lends credence to the statistical properties derived using the present dataset. This claim is also in accord with the recent high-quality imaging of NLSy1 galaxies by Olguín-Iglesias et al. (2020), as noted in Sect. 3.2.

The computed INOV DC and amplitudes $(\bar{\psi})$, using our 29 monitoring sessions devoted to the $15 \gamma$-ray NLSy1s, are listed in Table 4. To recall, these are based on the application of the $F_{e n h}$ and the $F^{\eta}$-tests, adopting a $99 \%$ confidence level threshold for confirming INOV detection (Sect. 4). The two tests have yielded INOV duty cycles of 39\% and 30\%, respectively, for our sample. The corresponding estimates using a photometric aperture radius of 3xFWHM (instead of 2xFWHM), for which the DLCs are noisier, are found to be $35 \%$ and $20 \%$, respectively. Thus, it is realistic to infer that the INOV DC for $\gamma$-ray NLSy1s is at least around $25 \%$, based 
on the $\mathrm{F}^{\eta}$-test and around $35 \%$ to $40 \%$ using the $F_{e n h}$-test. In a systematic study of INOV characteristics of several prominent classes of powerful AGN, based on the $F^{\eta}$-test with a confidence level threshold also set at 99\%, Goyal et al. (2013) showed that an INOV DC in excess of $20 \%$ is observed exclusively for blazar type objects, i.e., high-opticalpolarization quasars, BL Lacs, and TeV detected blazars (see, also Gopal-Krishna et al. 2011), their DC values being about $38 \%, 40 \%$ and $47 \%$, respectively. We may recall that, like the present campaign, the monitoring observations leading to these estimates were also made with 1-2 metre class telescopes and a detection threshold of the order of a few per cent was typically achieved. Thus, it seems that, as an AGN class, $\gamma$-ray NLSy1s are well matched to blazars in terms of the occurrence rate of INOV, even though their central engines operate in a regime of distinctly higher Eddington accretion rate, as inferred widely in the literature (see Sect. 1). One interesting consequence of the higher accretion rate for $\gamma$-ray NLSy1s is the expected enhancement of the thermal component of the AGN's optical emission, relative to its synchrotron emission (e.g., see Zhou et al. 2007; Paliya et al. 2014). Since the former is likely to be much less variable than the optical flux contributed by the (Doppler boosted) synchrotron jet, the fractional intranight optical variability (i.e., INOV amplitude $\psi$ ) would be suppressed due to the thermal contamination. The suppression can get compounded due to yet another contaminant, namely the steady optical emission coming from the host galaxy (if detected), which may be significant in the case of low redshift $(\mathrm{z} \lesssim 0.3) \gamma$ ray NLSy1s harbouring intrinsically much weaker jets than blazars. Some likely implications of both these contaminating/diluting processes have been quantitatively examined recently by Ojha et al. (2019), for the case of the prominent nearby $\gamma$-ray NLSy1 galaxy J032441.20+341045.0. The resulting prognosis in the present context is that future studies of $\gamma$-ray NLSy1s may well reveal an even stronger INOV, with a higher duty cycle, once it becomes possible to subtract out the contaminating thermal optical flux originating from the host galaxy and from the processes related to the AGN's inner accretion disc. Thus, it appears that the INOV activity in $\gamma$-ray NLSy1s may well turn out to be truly striking, even by the blazar standards.

We now turn attention to any sharp features present in the differential light curves, that are linked to the relativistic jet. In various intranight monitoring campaigns covering dozens of blazars, investigators have searched for brightness changes on time scales substantially shorter than an hour (e.g., Gopal-Krishna et al. 2011). Such ultra-short time scales are important since the implied physical size closely approaches the event horizon of a $10^{8}-10^{9} \mathrm{M}_{\odot}$ black hole (Wiita 2006, see, also, Armitage \& Reynolds 2003). Indeed, flux variability on minute-like time scales has been convincingly detected in $\gamma$-ray emission from some blazars, e.g., PKS 2155-304 (Aharonian et al. 2007) and PKS 1222+216 (Aleksić et al. 2011). However, several hundred intranight optical monitoring sessions targeting blazars have yielded just a few claims of INOV on the minute-like time scale and it is clear that such events are an extremely rare occurrence in blazar light curves (Gopal-Krishna \& Wiita 2018; Gopal-Krishna et al. 2019). A remarkable case of sharp optical variation, with an implied flux doubling time of just $~$ $1 \mathrm{hr}$ was observed in our monitoring of the $\gamma$-ray NLSy1 galaxy J032441.20+341045.0 on 2016-12-02 (see Fig. 1 for the DLCs). There we had argued that after allowing for the actual dilution by the thermal optical emission, the flux doubling time could be even shorter by a substantial margin, perhaps approaching the minute-like time scales (Ojha et al. 2019) observed in the $\gamma$-ray light curves of some blazars (see, above). In that study, we also pointed out that a similarly ultra-short flux doubling time can probably also be inferred from this AGN's already existing intranight DLCs dated 2012-12-09, published in Paliya et al. (2014) and, secondly, that such extremely rapid events may be correlated with the radio jet's superluminal speed (Ojha et al. 2019). Here we note that radio jets showing superluminal speeds have been shown to exist in 5 members of the present sample of 15 $\gamma$-ray NLSy1s. These five sources are: J032441.20+341045.0, $\mathrm{J} 122222.99+041315.9, \mathrm{~J} 150506.48+032630.8$, with $v_{\text {app }} / c$ of $9.1 \pm 0.3,0.9 \pm 0.3,1.1 \pm 0.4$, respectively (e.g., see Lister et al. 2016), and J084957.98+510829.0, J094857.32+002225.6 having $v_{a p p} / c$ of $6.6 \pm 0.8,9.1 \pm 0.3$, respectively (e.g., see Lister et al. 2019). Optical flux variability on minute to hourlike time scales has also been reported for these $5 \gamma$-ray NLSy1s (Liu et al. 2010; Paliya et al. 2013a, 2016; Ojha et al. 2019). The possibility of such a correlation could be firmly tested when the more extensive INOV database and superluminal speeds of the jets become available for $\gamma$-ray NLSy1s. In the present campaign, we have searched for additional clean examples of variability on time scales $\ll 1 \mathrm{hr}$ (see Figs. 1-4). As explained below, only two such events could be identified, although some more may well be found when DLCs with comparably dense sampling become available for our entire sample.

From Fig. 1, one of the two events is associated, once again, with J032441.20+341045.0 $(z=0.06)$, when roughly in the middle of its $3.4 \mathrm{hrs}$ long monitoring session on 2017-0104 , i.e., around 15:00 UT, its flux underwent a sharp drop by $\sim 3 \%$ within $\sim 6$ minutes and then, after remaining stagnant for $\sim 20$ minutes, the flux jumped back by $\sim 3 \%$ within 6 minutes. Now, following the arguments made by us previously in Ojha et al. (2019) and recalled earlier in this section, the actual amplitude of both these flux changes are likely to be several times greater once a correction is made for the dilution of the jet's optical emission, due to the (much steadier) thermal emission contributed by this strongly accreting AGN itself and also by its host galaxy. It is also interesting to note that out of the total 10 monitoring sessions of $>3 \mathrm{hr}$ duration, reported for this AGN here (5 sessions) and in previous studies (Paliya et al. 2013a, 2014; Ojha et al. 2019), strong features of duration $\ll 1 \mathrm{hr}$ have been observed in the DLCs of as many as 3 sessions. Thus, large and ultra-fast brightness changes appear to be a remarkable behavioural signature of this $\gamma$-ray NLSy1 galaxy! Moving now to the second large event of comparable sharpness, found in the present dataset, it can be seen in the DLCs of the source J094857.32+002225.8 $(z=0.584)$ on $2017-12-21$. During that 5.2 hrs long monitoring session (Fig. 2), at around 19.55 UT, the flux showed a sharp jump (between two consecutive points) of $\sim 7 \%$ within $\sim 15$ minutes. Although, for this source, no estimates are currently available for the dilution by thermal optical emission, such a contamination is quite likely (especially from the AGN accretion disc), going by the above-mentioned example of the $\gamma$-ray NLSy1 J032441.20+341045.0. Note that a very strong INOV of J094857.32+002225.8 has been re- 
ported previously in the independent campaigns conducted by Liu et al. (2010) and Maune et al. (2013). The most rapid flux evolution, found by the latter team, occurred on 201104-01 and amounted to a rate of $0.2-0.3 \mathrm{mag} \mathrm{hr}^{-1}$. Thus, both these $\gamma$-ray NLSy1s are proto-types of blazar-like jet activity, probably even surpassing them in the rapidity of brightness change. It is also interesting to note that these two AGN, although grossly different in redshift, display highly superluminal nuclear radio jets, with a speed of up to $7 \mathrm{c}$ in case of J032441.20+341045.0 (Fuhrmann et al. 2016) and 9c for J094857.32+002225.6 (Lister et al. 2019). Thus, in summary, the fact that in the present large and unbiased sample of $\gamma$-ray NLSy1s, at least 2 out of total 29 sessions have an event with optical flux doubling time of $\lesssim 1 \mathrm{hr}$. In contrast, such rapid optical variations have hardly ever been observed in the extensive monitoring programs targetting blazars (see above). Could the contrast be a reflection of the qualitative physical differences between the central engines of these two types of jetted AGNs (Sect. 1)? This appears to be a potentially important emerging clue, to be probed by further observations.

\section{CONCLUSIONS}

Although the first INOV observations of a $\gamma$-ray NLSy1 galaxy were reported a decade ago (Liu et al. 2010), subsequent follow up has been limited to just 6 members of this intriguing class of AGN. The present study, based on a much larger sample (see below) is the first attempt to systematically characterise the INOV properties of $\gamma$-ray NLSy1 galaxies. These jetted AGNs are particularly interesting since their central engines are thought to operate in a physical regime of accretion rate different from what is believed to occur in (more powerful) blazars. Our study is based on an unbiased sample of $15 \gamma$-ray NLSy1s, assembled by applying well-defined selection criteria to a total of 20 such objects known at present. Based on their monitoring in 36 sessions of minimum 3 hrs duration, we estimate an INOV duty cycle of 25 - 30 per cent for a typical INOV amplitude detection threshold of around $3-5 \%$. Among the powerful AGNs, only blazars are known to achieve INOV DC exceeding $\sim 20 \%$. Thus, as a class, $\gamma$-ray NLSy1s resemble blazars even in INOV properties. As discussed in Sect. 5, it is quite likely that we may have underestimated the DC for $\gamma$-ray NLSy1s because their nonthermal optical emission which is primarily responsible for INOV, could be significantly diluted by the much less variable optical emission contributed by the host galaxy (in case of lower redshift sources) and the accretion disc operating at a high Eddington accretion rate (Boroson \& Green 1992; Pounds et al. 1995; Sulentic et al. 2000; Boroson 2002; Collin \& Kawaguchi 2004; Grupe \& Mathur 2004; Paliya 2019; Ojha et al. 2020a).

Further, we have detected in the light curves of two prominent members of our sample of $15 \gamma$-ray NLSy1s, ultrarapid brightness changes amounting to a clear level change within $\sim 0.1 \mathrm{hr}$. Quite plausibly, with a similarly dense sampling of the light curves for our entire sample, more such events would be found. But already, this result seems to stand in contrast to the observed extreme rarity of such distinct ultra-rapid events of optical variability in blazar light curves, even though they vastly outnumber the light curves currently available for $\gamma$-ray NLSy1s. If this difference is firmly established by further observations of $\gamma$-ray NLSy1s, that would provide a useful input to the models of jet formation in these enigmatic AGNs.

\section{ACKNOWLEDGEMENTS}

We thank an anonymous referee for his/her very important comments that helped us to improve this manuscript considerably. We also thank the concerned ARIES staff for assistance during the observations. G-K thanks the Indian National Science Academy for a Senior Scientist fellowship.

\section{DATA AVAILABILITY}

The data used in this article are from 1.3-m Devasthal Fast Optical Telescope (DFOT) of Aryabhatta Research Institute of Observational Sciences (ARIES), Nainital, India (https:/ / www.aries.res.in/facilities/astronomicaltelescopes/13m-telescope), which will be shared on reasonable request to the corresponding author.

\section{REFERENCES}

Abdo A. A. et al., 2009a, ApJ, 699, 976

Abdo A. A. et al., 2009b, ApJ, 707, 727

Abdo A. A. et al., 2009c, ApJ, 707, L142

Abdollahi S. et al., 2020, ApJS, 247, 33

Abolfathi B. et al., 2018, ApJS, 235, 42

Aharonian F. et al., 2007, ApJ, 664, L71

Aleksić J. et al., 2011, ApJ, 730, L8

Armitage P. J., Reynolds C. S., 2003, MNRAS, 341, 1041

Bai J. M., Xie G. Z., Li K. H., Zhang X., Liu W. W., 1999, A\&AS, 136, 455

Berton M. et al., 2018, A\&A, 614, A87

Boller T., Brandt W. N., Fink H., 1996, A\&A, 305, 53

Boroson T. A., 2002, ApJ, 565, 78

Boroson T. A., Green R. F., 1992, ApJS, 80, 109

Calderone G., Ghisellini G., Colpi M., Dotti M., 2013, MNRAS, 431, 210

Camenzind M., Krockenberger M., 1992, A\&A, 255, 59

Carini M. T., 1990, PhD thesis, , George State University, Atlanta, USA, (1990)

Carini M. T., Miller H. R., Noble J. C., Goodrich B. D., 1992, AJ, 104, 15

Carini M. T., Noble J. C., Miller H. R., 2003, AJ, 125, 1811

Carini M. T., Noble J. C., Taylor R., Culler R., 2007, AJ, 133, 303

Cellone S. A., Romero G. E., Araudo A. T., 2007, MNRAS, 374,357

Cellone S. A., Romero G. E., Combi J. A., 2000, AJ, 119, 1534

Chakrabarti S. K., Wiita P. J., 1993, ApJ, 411, 602

Chand H., Kumar P., Gopal-Krishna, 2014, MNRAS, 441, 726

Ciprini S., Fermi-LAT Collaboration, 2018, in Revisiting Narrow-Line Seyfert 1 Galaxies and their Place in the Universe, p. 20

Collin S., Kawaguchi T., 2004, A\&A, 426, 797

Cracco V., Ciroi S., Berton M., Di Mille F., Foschini L., La Mura G., Rafanelli P., 2016, MNRAS, 462, 1256 

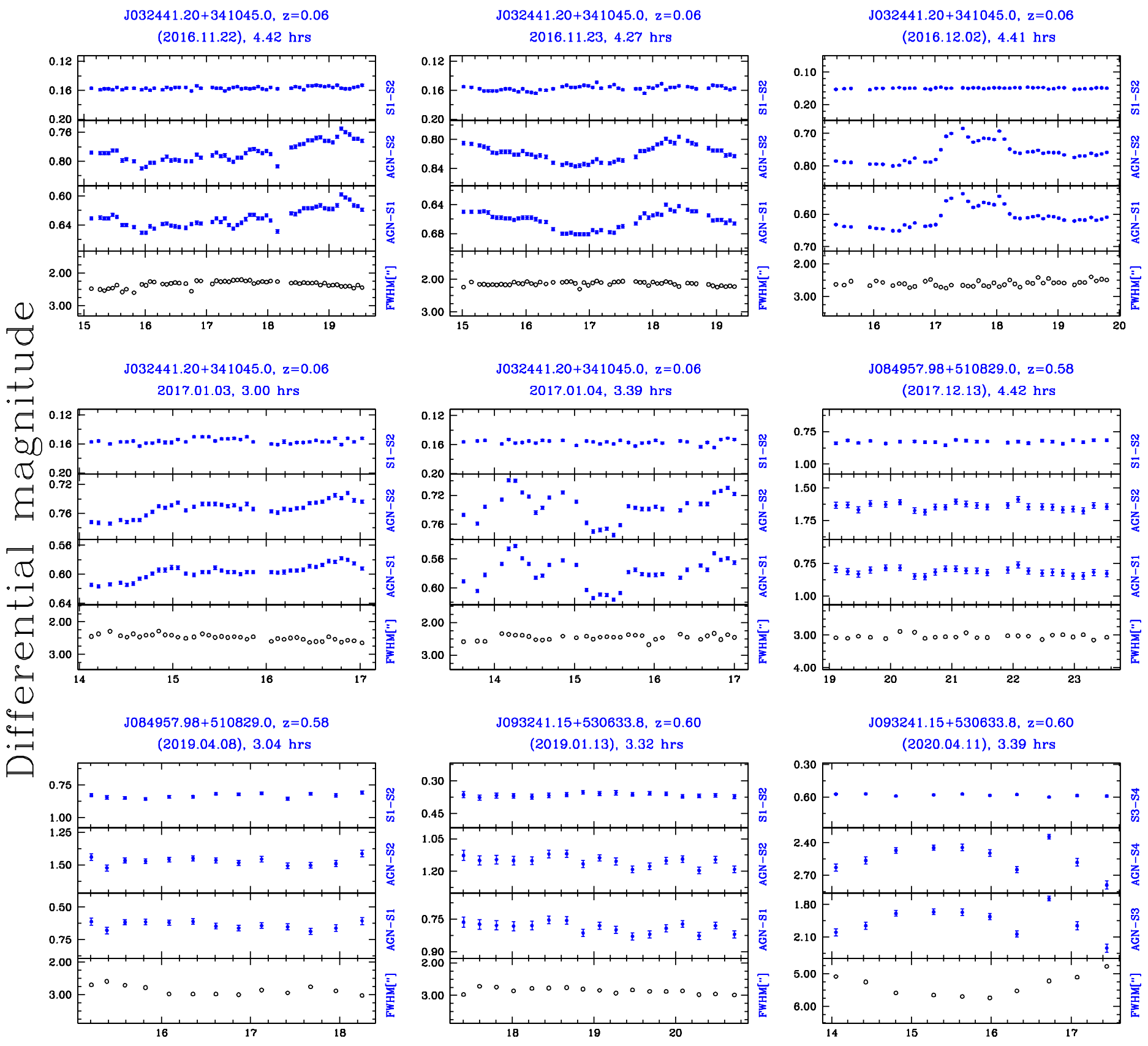

(2017.12.13), $4.42 \mathrm{hrs}$

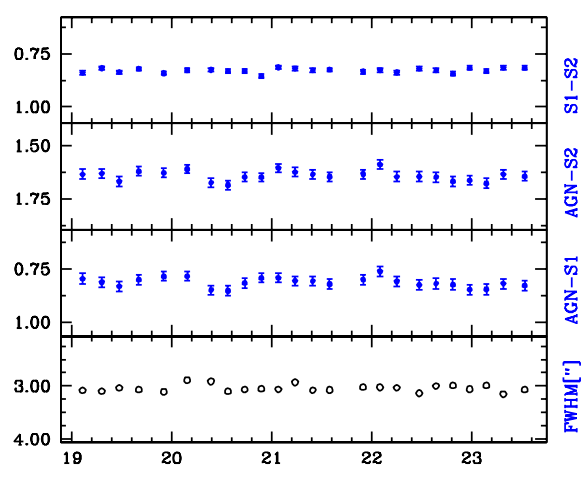

J09324 1.15+530633.8, $\mathrm{z}=0.60$

J093241.15+530633.8, $\mathrm{z}=0.60$ (2019.01.13), $3.32 \mathrm{hrs}$

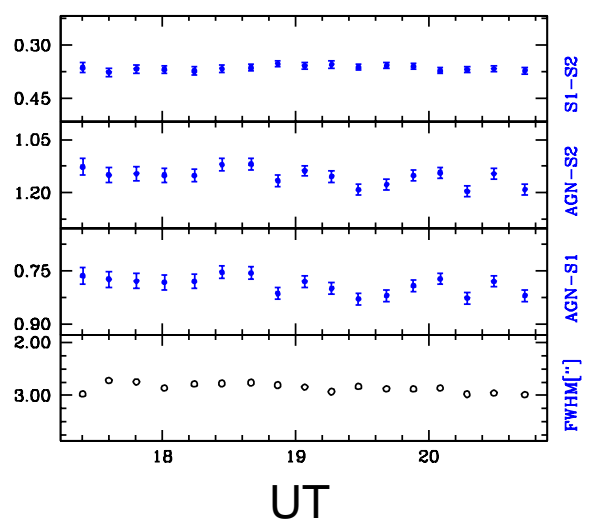

(2020.04.11), $3.39 \mathrm{hrs}$

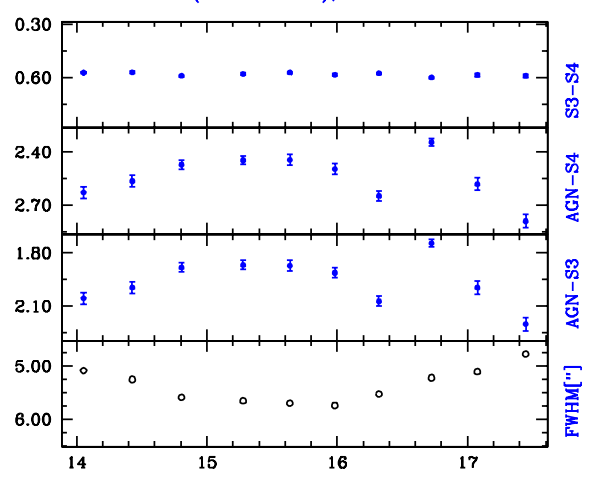

Figure 1. Intranight differential light curves (DLCs) of the first $3 \gamma$-ray NLSy1s from our sample of $15 \gamma-$ ray NLSy1s. The AGN name, its redshift, and some observational details are given at the top of each panel. The sessions with the date given inside parentheses at the top of each panel were taken for the statistical analysis. In each panel, the upper DLC is derived using the chosen two (non-varying) comparison stars, while the lower two DLCs are the 'NLSy1-star' DLCs, as defined in the labels on the right side. The bottom panel displays the variations of the seeing disc (FWHM) during the monitoring session. 

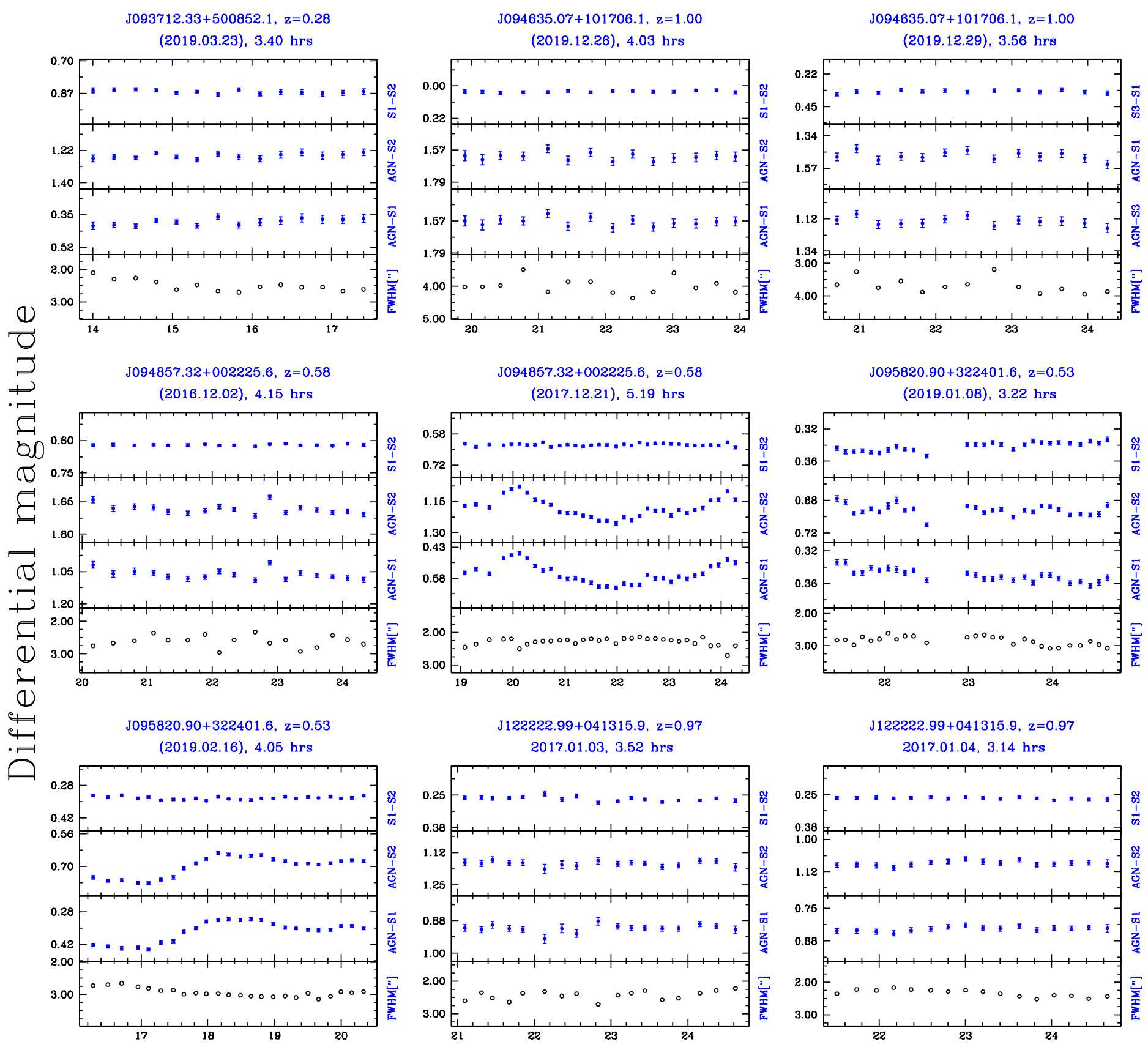

$(2017.12 .21), 5.19 \mathrm{hrs}$

(2019.01.08), $3.22 \mathrm{hrs}$
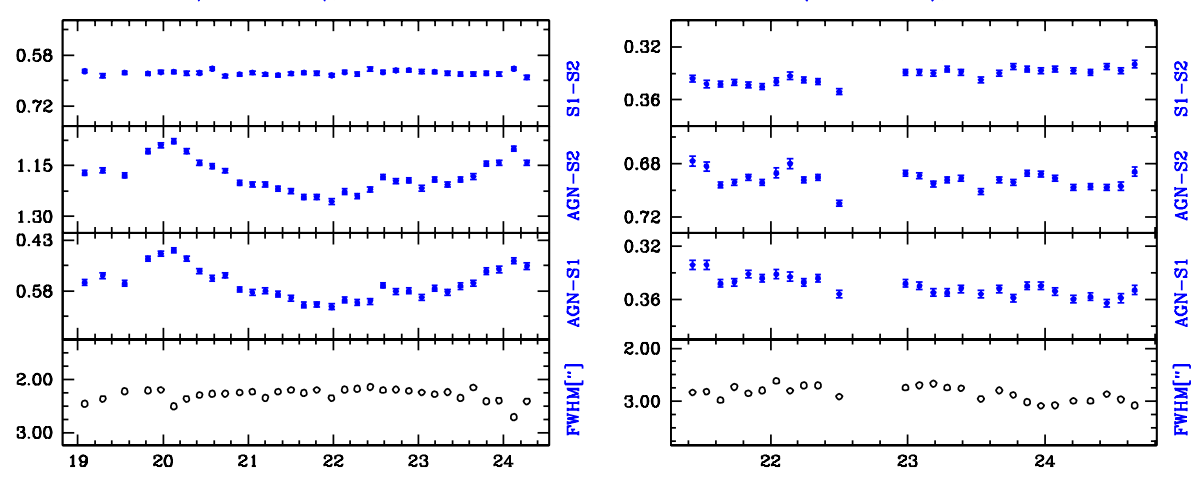

$\mathrm{J} 122222.99+041315.9, \mathrm{z}=0.97$ $2017.01 .03,3.52 \mathrm{hrs}$

J122222.99+041315.9, $\mathrm{z}=0.97$
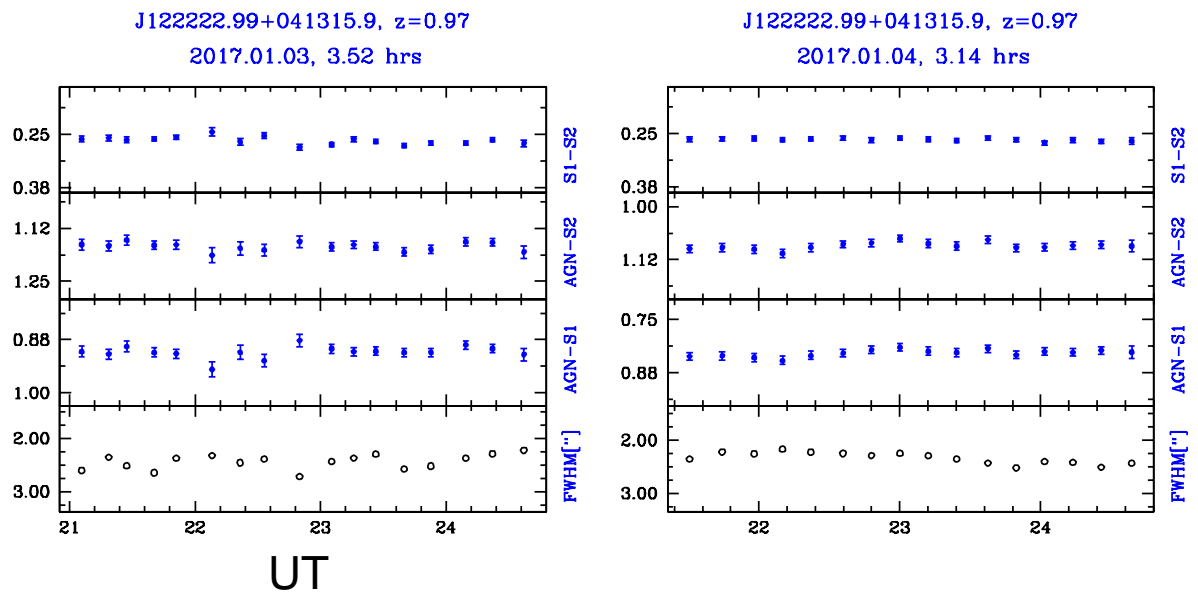

Figure 2. Same as Fig. 1, but for the next $5 \gamma$-ray NLSy1s from our sample of $15 \gamma$-ray NLSy1s galaxies.

Czerny B., Siemiginowska A., Janiuk A., Gupta A. C., 2008, MNRAS, 386, 1557

D'Ammando F. et al., 2012, MNRAS, 426, 317

D'Ammando F., Orienti M., Larsson J., Giroletti M., 2015, MNRAS, 452, 520

de Diego J. A., 2010, AJ, 139, 1269 de Diego J. A., 2014, AJ, 148, 93

Decarli R., Dotti M., Fontana M., Haardt F., 2008, MNRAS, 386, L15

Deo R. P., Crenshaw D. M., Kraemer S. B., 2006, AJ, 132, 321

Fan J. H., Qian B. C., Tao J., 2001, A\&A, 369, 758

Ferrara E. C., Miller H. R., McFarland J. P., Williams A. M., 

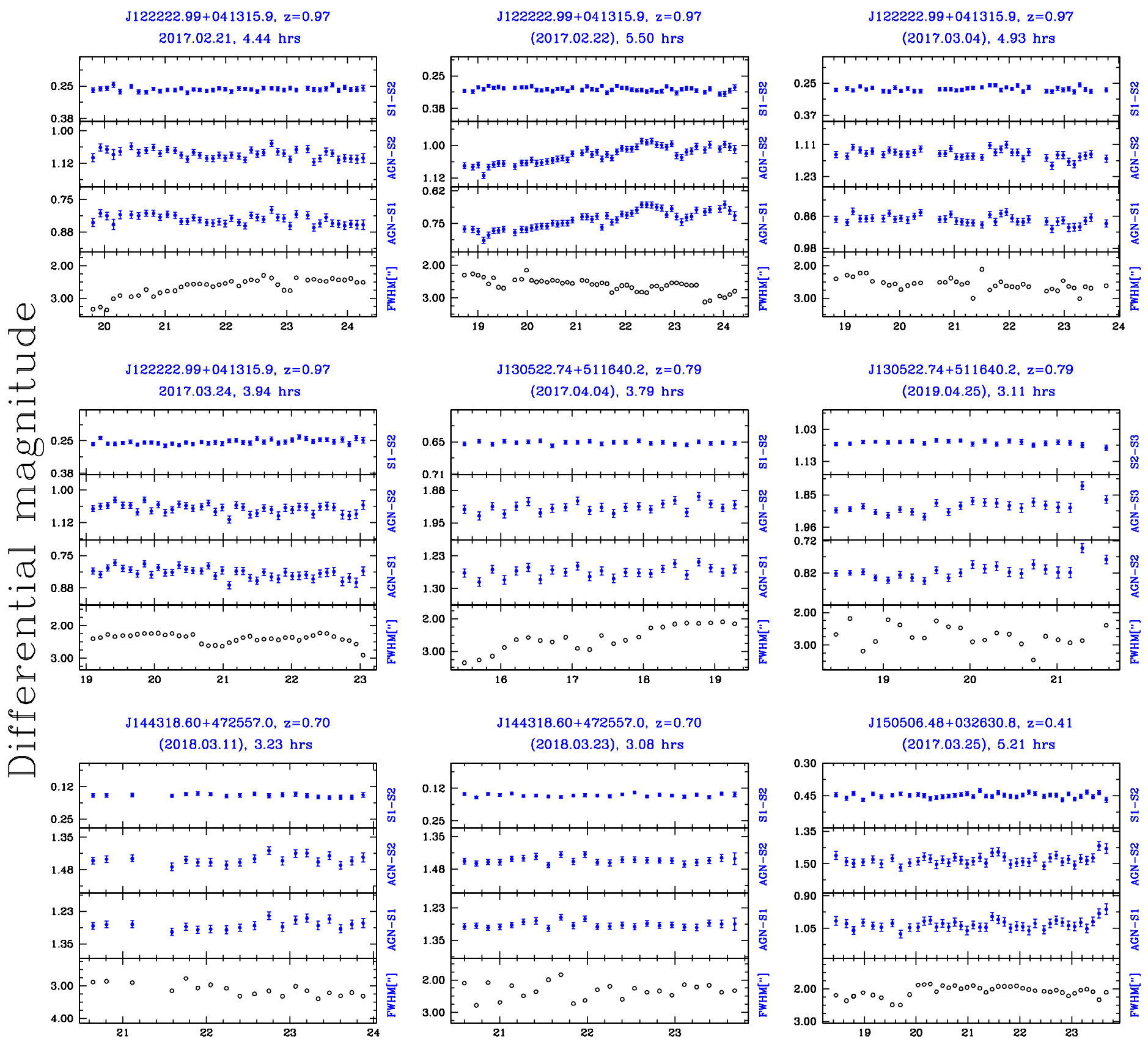

(2017.04.04), $3.79 \mathrm{hrs}$

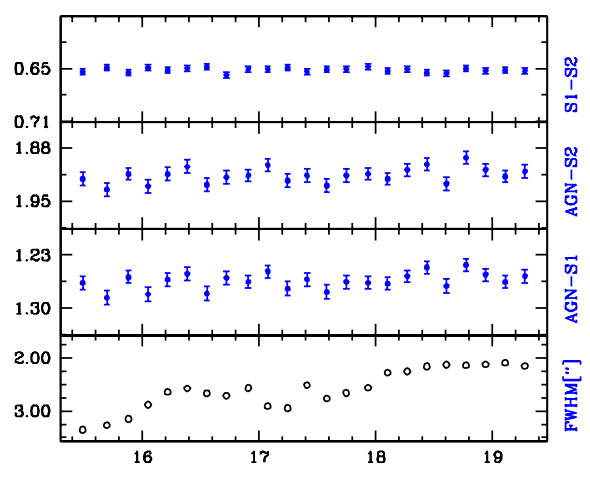

(2019.04.25), $3.11 \mathrm{hrs}$

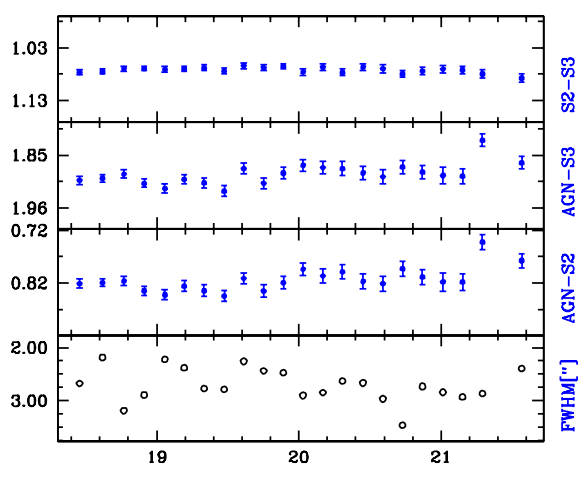

$\mathrm{J} 144318.60+472557.0, \mathrm{z}=0.70$

$\mathrm{J} 150506.48+032630.8, \mathrm{z}=0.41$ (2018.03.23), $3.08 \mathrm{hrs}$

(2017.03.25), $5.21 \mathrm{hrs}$
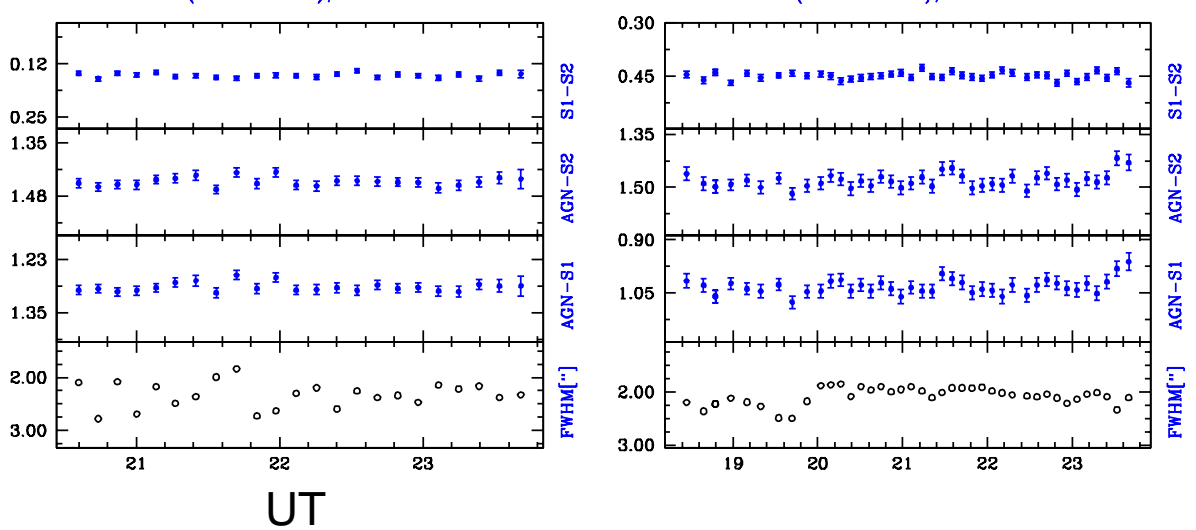

Figure 3. Same as Fig. 1, but for the next $3 \gamma$-ray NLSy1s from our sample of $15 \gamma$-ray NLSy1s galaxies.

Wilson J. W., Fried R. E., Noble J. C., 2001, in Astronomical Society of the Pacific Conference Series, Vol. 224, Probing the Physics of Active Galactic Nuclei, Peterson B. M., Pogge R. W., Polidan R. S., eds., p. 319

Foschini L., 2011, in Narrow-Line Seyfert 1 Galaxies and their Place in the Universe, p. 24
Foschini L. et al., 2015, A\&A, 575, A13

Foschini L., Fermi/Lat Collaboration, Ghisellini G., Maraschi L., Tavecchio F., Angelakis E., 2010, in Astronomical Society of the Pacific Conference Series, Vol. 427, Accretion and Ejection in AGN: a Global View, Maraschi L., Ghisellini G., Della Ceca R., Tavecchio F., eds., pp. 

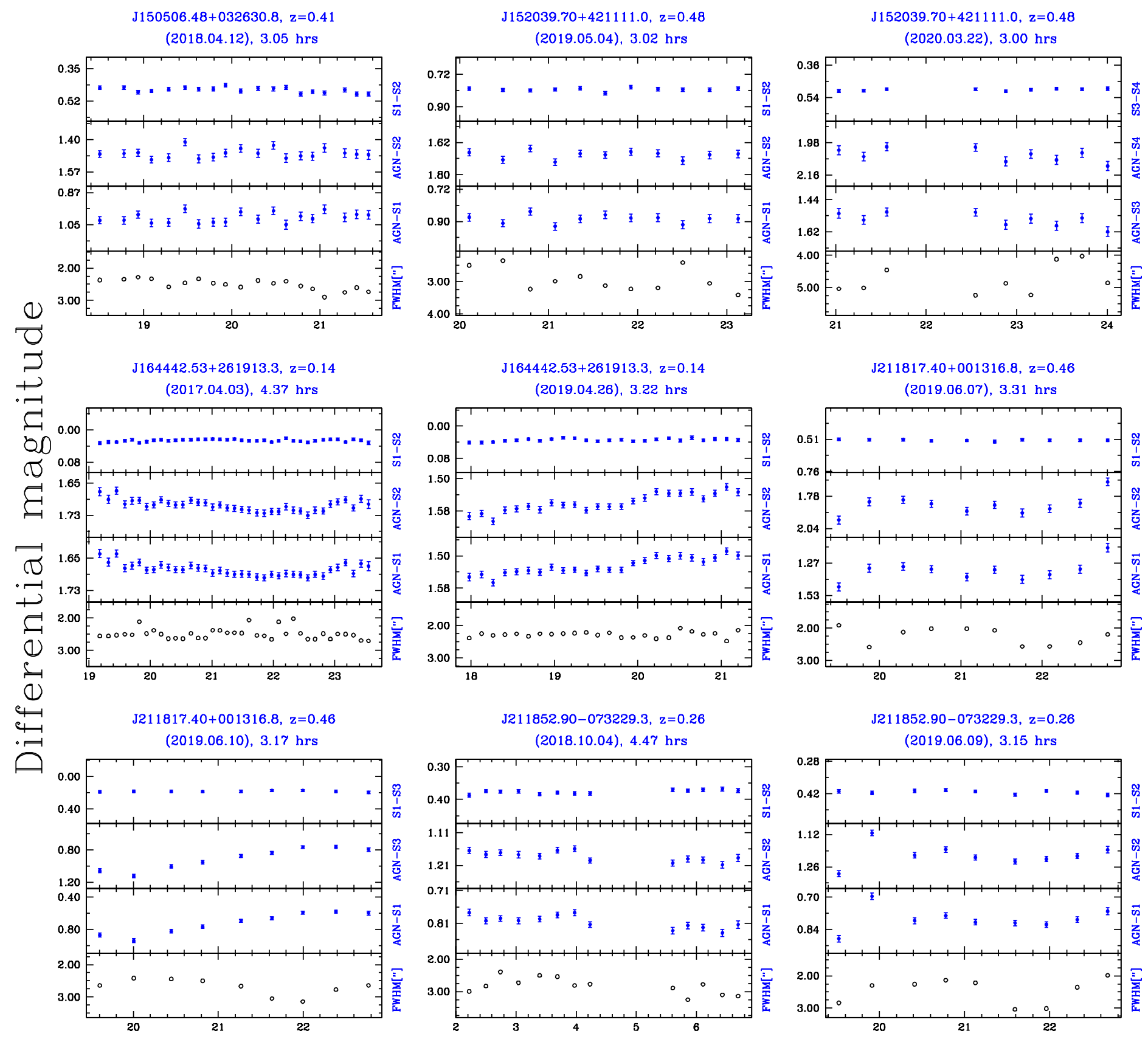

(2019.04.26), $3.22 \mathrm{hrs}$

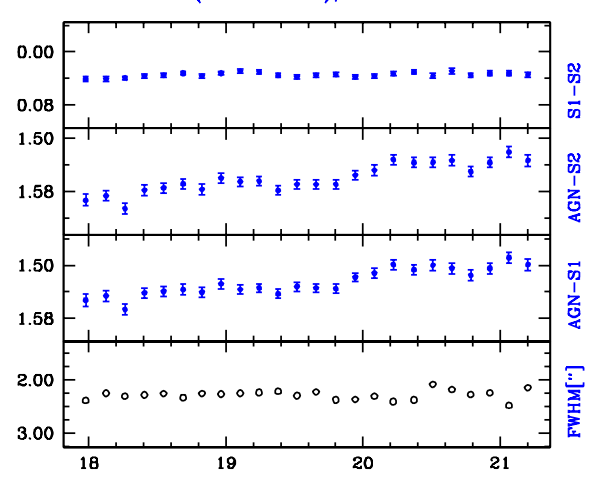

(2019.06.07), $3.31 \mathrm{hrs}$

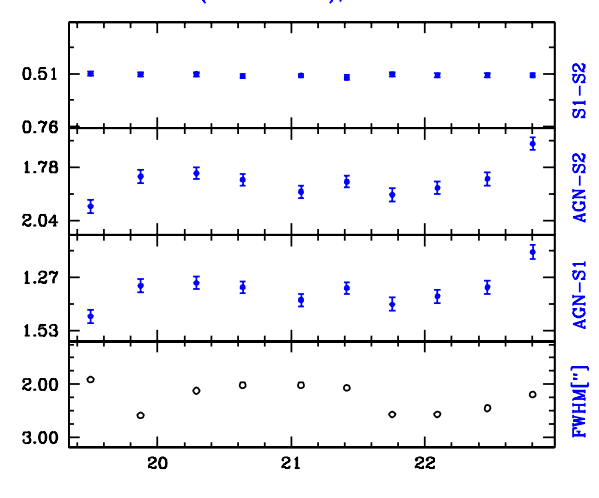

J21 1852.90-073229.3, $\mathrm{z}=0.26$

J211852.90-073229.3, $\mathrm{z}=0.26$

(2018.10.04), $4.47 \mathrm{hrs}$

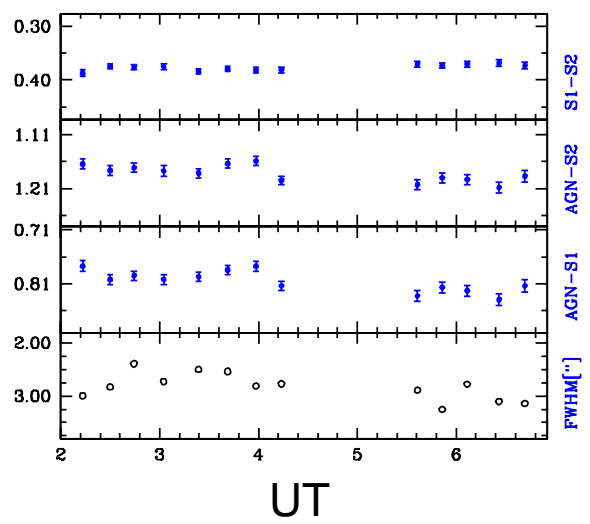

(2019.06.09), $3.15 \mathrm{hrs}$

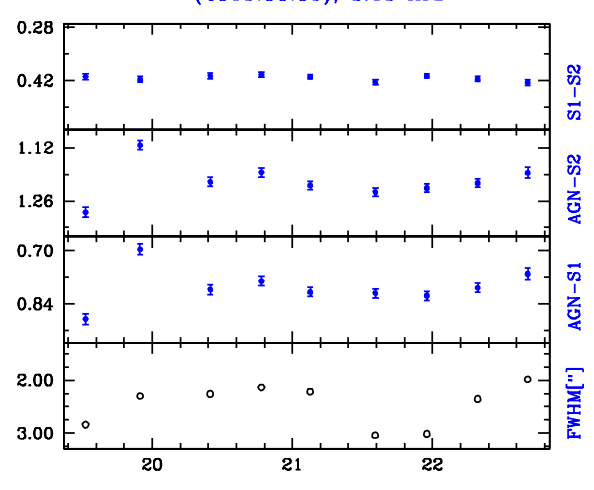

Figure 4. Same as Fig. 1, but for the last $4 \gamma$-ray NLSy1s from our sample of $15 \gamma$-ray NLSy1s galaxies.

243-248

Fraix-Burnet D., Marziani P., D'Onofrio M., Dultzin D., 2017, Frontiers in Astronomy and Space Sciences, 4, 1

Fuhrmann L. et al., 2016, Research in Astronomy and Astrophysics, 16, 176

Ganci V., Marziani P., D’Onofrio M., del Olmo A., Bon E.,
Bon N., Negrete C. A., 2019, A\&A, 630, A110

Goodrich R. W., Stringfellow G. S., Penrod G. D., Filippenko A. V., 1989, ApJ, 342, 908

Gopal-Krishna, Britzen S., Wiita P., 2019, Bulletin de la Societe Royale des Sciences de Liege, 88, 132

Gopal-Krishna, Goyal A., Joshi S., Karthick C., Sagar R., Wi- 
ita P. J., Anupama G. C., Sahu D. K., 2011, MNRAS, 416, 101

Gopal-Krishna, Joshi R., Chand H., 2013, MNRAS, 430, 1302 Gopal-Krishna, Sagar R., Wiita P. J., 1995, MNRAS, 274, 701 Gopal-Krishna, Stalin C. S., Sagar R., Wiita P. J., 2003, ApJ, 586, L25

Gopal-Krishna, Wiita P. J., 1992, A\&A, 259, 109

Gopal-Krishna, Wiita P. J., 2018, Bulletin de la Societe Royale des Sciences de Liege, 87, 281

Gopal-Krishna, Wiita P. J., Altieri B., 1993, A\&A, 271, 89

Goyal A., Gopal-Krishna, Wiita P. J., Anupama G. C., Sahu D. K., Sagar R., Joshi S., 2012, A\&A, 544, A37

Goyal A., Gopal-Krishna, Paul J. W., Stalin C. S., Sagar R., 2013, MNRAS, 435, 1300

Grupe D., Beuermann K., Thomas H.-C., Mannheim K., Fink H. H., 1998, A\&A, 330, 25

Grupe D., Mathur S., 2004, ApJ, 606, L41

Gupta A. C., Joshi U. C., 2005, A\&A, 440, 855

Heidt J., Wagner S. J., 1996, A\&A, 305, 42

Howell S. B., 1989, PASP, 101, 616

Howell S. B., Warnock, III A., Mitchell K. J., 1988, AJ, 95, 247

Hughes P. A., Aller H. D., Aller M. F., 1991, ApJ, 374, 57

Itoh R. et al., 2013, ApJ, 775, L26

Jang M., Miller H. R., 1995, ApJ, 452, 582

Joshi R., Chand H., Gupta A. C., Wiita P. J., 2011, MNRAS, 412,2717

Kellermann K. I., Condon J. J., Kimball A. E., Perley R. A., Ivezić Ž., 2016, ApJ, 831, 168

Kellermann K. I., Sramek R., Schmidt M., Shaffer D. B., Green R., 1989, AJ, 98, 1195

Kellermann K. I., Sramek R. A., Schmidt M., Green R. F., Shaffer D. B., 1994, AJ, 108, 1163

Klimek E. S., Gaskell C. M., Hedrick C. H., 2004, ApJ, 609, 69

Komossa S., 2018, in Revisiting Narrow-Line Seyfert 1 Galaxies and their Place in the Universe, p. 15

Komossa S., Meerschweinchen J., 2000, A\&A, 354, 411

Komossa S., Voges W., Xu D., Mathur S., Adorf H.-M., Lemson G., Duschl W. J., Grupe D., 2006, AJ, 132, 531

Kshama S. K., Paliya V. S., Stalin C. S., 2017, MNRAS, 466, 2679

Kumar P., Chand H., Gopal-Krishna, 2016, MNRAS, 461, 666

Kumar P., Gopal-Krishna, Chand H., 2015, MNRAS, 448, 1463

Kumar P., Gopal-Krishna, Stalin C. S., Chand H., Srianand R., Petitjean P., 2017, MNRAS, 471, 606

Leighly K. M., 1999, ApJS, 125, 297

Lister M., 2018, in Revisiting Narrow-Line Seyfert 1 Galaxies and their Place in the Universe, p. 22

Lister M. L. et al., 2016, AJ, 152, 12

Lister M. L. et al., 2019, ApJ, 874, 43

Liu H., Wang J., Mao Y., Wei J., 2010, ApJ, 715, L113

Mangalam A. V., Wiita P. J., 1993, ApJ, 406, 420

Marconi A., Axon D. J., Maiolino R., Nagao T., Pastorini G., Pietrini P., Robinson A., Torricelli G., 2008, ApJ, 678, 693

Marscher A. P., 1996, in Astronomical Society of the Pacific Conference Series, Vol. 110, Blazar Continuum Variability,

Miller H. R., Webb J. R., Noble J. C., eds., p. 248

Marscher A. P., Gear W. K., 1985, ApJ, 298, 114

Mathur S., 2000, MNRAS, 314, L17

Mathur S., Kuraszkiewicz J., Czerny B., 2001, New A, 6, 321
Maune J. D., Miller H. R., Eggen J. R., 2013, ApJ, 762, 124

Miller H. R., Carini M. T., Goodrich B. D., 1989, Nature, 337, 627

Miller H. R., Ferrara E. C., McFarland J. P., Wilson J. W., Daya A. B., Fried R. E., 2000, New A Rev., 44, 539

Monet D. G., 1998, in Bulletin of the American Astronomical Society, Vol. 30, American Astronomical Society Meeting Abstracts, p. 1427

Ojha V., Chand H., Dewangan G. C., Rakshit S., 2020a, ApJ, 896, 95

Ojha V., Chand H., Gopal-Krishna, 2018, Bulletin de la Societe Royale des Sciences de Liege, 87, 387

Ojha V., Chand H., Gopal-Krishna, Mishra S., Chand K., 2020b, MNRAS, 493, 3642

Ojha V., Gopal-Krishna, Chand H., 2019, MNRAS, 483, 3036

Olguín-Iglesias A., Kotilainen J., Chavushyan V., 2020, MNRAS, 492, 1450

Osterbrock D. E., Pogge R. W., 1985, ApJ, 297, 166

Paliya V. S., 2019, Journal of Astrophysics and Astronomy, 40, 39

Paliya V. S., Ajello M., Rakshit S., Mandal A. K., Stalin C. S., Kaur A., Hartmann D., 2018, ApJ, 853, L2

Paliya V. S., Parker M. L., Jiang J., Fabian A. C., Brenneman L., Ajello M., Hartmann D., 2019, ApJ, 872, 169

Paliya V. S., Rajput B., Stalin C. S., Pandey S. B., 2016, ApJ, 819,121

Paliya V. S., Sahayanathan S., Parker M. L., Fabian A. C., Stalin C. S., Anjum A., Pandey S. B., 2014, ApJ, 789, 143

Paliya V. S., Stalin C. S., Kumar B., Kumar B., Bhatt V. K., Pandey S. B., Yadav R. K. S., 2013a, MNRAS, 428, 2450

Paliya V. S., Stalin C. S., Shukla A., Sahayanathan S., 2013b, ApJ, 768, 52

Peterson B. M., 2011, in Narrow-Line Seyfert 1 Galaxies and their Place in the Universe, p. 32

Peterson B. M. et al., 2000, ApJ, 542, 161

Pogge R. W., 2011, in Narrow-Line Seyfert 1 Galaxies and their Place in the Universe, p. 2

Pounds K. A., Done C., Osborne J. P., 1995, MNRAS, 277, L5 Qian S. J., Quirrenbach A., Witzel A., Krichbaum T. P., Hummel C. A., Zensus J. A., 1991, A\&A, 241, 15

Rakshit S., Stalin C. S., Chand H., Zhang X.-G., 2017, ApJS, 229, 39

Ramírez A., de Diego J. A., Dultzin D., González-Pérez J.-N., 2009, AJ, 138, 991

Romero G. E., Cellone S. A., Combi J. A., 1999, A\&AS, 135, 477

Sagar R., Kumar B., Omar A., Pandey A. K., 2010, in Astronomical Society of India Conference Series, Vol. 1, Astronomical Society of India Conference Series

Shuder J. M., Osterbrock D. E., 1981, ApJ, 250, 55

Singh V., Chand H., 2018, MNRAS, 480, 1796

Stalin C. S., Gopal-Krishna, Sagar R., Wiita P. J., 2004, MNRAS, 350, 175

Stetson P. B., 1987, PASP, 99, 191

Stetson P. B., 1992, in Astronomical Society of the Pacific Conference Series, Vol. 25, Astronomical Data Analysis Software and Systems I, Worrall D. M., Biemesderfer C., Barnes J., eds., p. 297

Stocke J. T., Morris S. L., Weymann R. J., Foltz C. B., 1992, ApJ, 396, 487

Sulentic J. W., Zwitter T., Marziani P., Dultzin-Hacyan D., 2000, ApJ, 536, L5 
Ulrich M.-H., Maraschi L., Urry C. M., 1997, ARA\&A, 35, 445

Urry C. M., Padovani P., 1995, PASP, 107, 803

Visnovsky K. L., Impey C. D., Foltz C. B., Hewett P. C., Weymann R. J., Morris S. L., 1992, ApJ, 391, 560

Viswanath G., Stalin C. S., Rakshit S., Kurian K. S., Ujjwal K., Gudennavar S. B., Kartha S. S., 2019, ApJ, 881, L24

Wagner S. J., Witzel A., 1995, ARA\&A, 33, 163

Wang T., Brinkmann W., Bergeron J., 1996, A\&A, 309, 81

Wiita P. J., 2006, in Astronomical Society of the Pacific Conference Series, Vol. 350, Blazar Variability Workshop II: Entering the GLAST Era, Miller H. R., Marshall K., Webb J. R., Aller M. F., eds., p. 183

Yang H. et al., 2018, MNRAS, 477, 5127

Yao S., Komossa S., Liu W.-J., Yi W., Yuan W., Zhou H., Wu X.-B., 2019, MNRAS, 487, L40

Yao S., Yuan W., Zhou H., Komossa S., Zhang J., Qiao E., Liu B., 2015, MNRAS, 454, L16

Yuan W., Zhou H. Y., Komossa S., Dong X. B., Wang T. G., Lu H. L., Bai J. M., 2008, ApJ, 685, 801

Zensus J. A., 1997, ARA\&A, 35, 607

Zhou H., Wang T., Yuan W., Lu H., Dong X., Wang J., Lu Y., 2006, ApJS, 166, 128

Zhou H. et al., 2007, ApJ, 658, L13

Zhou H.-Y., Wang T.-G., 2002, Chinese J. Astron. Astrophys., 2,501 